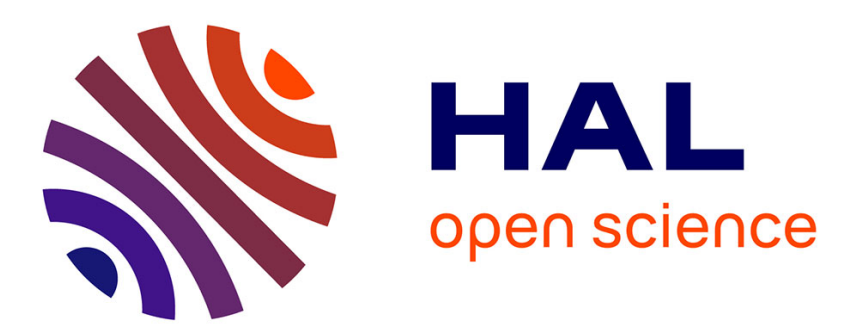

\title{
Uzawa Block Relaxation Domain Decomposition Method for the Two-Body Contact Problem With Tresca Friction Jonas Koko
}

\section{To cite this version:}

Jonas Koko. Uzawa Block Relaxation Domain Decomposition Method for the Two-Body Contact Problem With Tresca Friction. Computer Methods in Applied Mechanics and Engineering, 2008, 198 (3-4), pp.Pages 420-431. 10.1016/j.cma.2008.08.011 . hal-00678467

\section{HAL Id: hal-00678467 https://hal.science/hal-00678467}

Submitted on 13 Mar 2012

HAL is a multi-disciplinary open access archive for the deposit and dissemination of scientific research documents, whether they are published or not. The documents may come from teaching and research institutions in France or abroad, or from public or private research centers.
L'archive ouverte pluridisciplinaire HAL, est destinée au dépôt et à la diffusion de documents scientifiques de niveau recherche, publiés ou non, émanant des établissements d'enseignement et de recherche français ou étrangers, des laboratoires publics ou privés. 


\title{
Uzawa Block Relaxation Domain Decomposition Method for the Two-Body Contact Problem With Tresca Friction
}

\author{
Jonas $\mathrm{KOKO}^{1}$ \\ Research Report LIMOS/RR-08-01
}

15 janvier 2008

${ }^{1}$ LIMOS, Université Blaise Pascal - CNRS UMR 6158, Campus des Cézeaux B.P. 10125, 63173 Aubière cedex, France; e-mail: koko@isima.fr 


\begin{abstract}
We propose a Uzawa block relaxation domain decomposition method for a two-body contact problem with Tresca friction. We introduce auxiliary interface unknowns to transform the variational problem into a saddle-point problem. Applying a Uzawa block relaxation algorithm to the corresponding augmented Lagrangian functional we obtain a domain decomposition algorithm in which we have to solve two uncoupled linear elasticity subproblems in each iteration. The auxiliary unknowns are computed explicitly using Kuhn-Tucker conditions and Fenchel duality theory. Numerical experiments show the scalability of the domain decomposition algorithm on matching or nonmatching meshes for two- or three-dimensional contact problems.
\end{abstract}

Keywords: Two-body contact, augmented Lagrangian, Kuhn-Tucker conditions, Fenchel duality, domain decomposition 


\section{Introduction}

Various domain decomposition methods, for contact problems, have been proposed by many authors in order to speed up the solution. Dostal et al. [7] have proposed an augmented Lagrangian/domain decomposition method. The scalability of the method has been studied by Schöberl [28], Dureisseix and Farhat [9], Dostál and Horák [6]. The methods presented in $[9,7,6,28]$ are based on the FETI domain decomposition method [11] and designed on the discrete level. Kosior et al. [23] have proposed a domain decomposition method based on boundary element techniques. Bayada et al. have proposed Neumann-Neumann [2] and a Neumann-Dirichlet [1] domain decomposition algorithms. Koko [20] has proposed a domain decomposition method based on the decomposition of the set of admissible displacements introduced by the nonpenetration condition. Note that the domain decomposition proposed in $[1,2,20]$ are designed on the continuous problem.

We propose in this paper an augmented Lagrangian based domain decomposition method for the two-body contact problem with Tresca friction, designed on the continuous level. Auxiliary unknowns are introduced to separate the subdomains representing the linear elastic bodies, leading to a constrained (convex) minimization formulation. Applying a Uzawa block relaxation method to the corresponding augmented Lagrangian functional yields a domain decomposition method in which we have to solve two uncoupled linear elasticity subproblems in every iteration. The auxiliary unknowns are computed explicitly using Kuhn-Tucker and Fenchel duality. The domain decomposition presented in this article is an operator-splitting type method $[12,13]$. We refer to [5] for block relaxation method in convex minimization and to [26] (and references therein) for Lagrange multiplier based domain decomposition methods. The Tresca friction problem is a commonly chosen approach towards the solution of the Coulomb frictional contact problem through fixed-point procedures (see e.g. [8, 15, 24]).

The paper is organized as follows. In Section 2 we state the two-body contact problem with Tresca friction. The Uzawa block relaxation domain decomposition algorithm is described in Section 3 followed by the convergence results in Section 4. In Section 5 we report numerical experiments carried out with the new algorithm.

\section{Problem statement}

We consider two elastic bodies each of them occupying a bounded domain $\Omega^{\alpha}$ in $\mathbb{R}^{d}$, $d \geq 2$ and $\alpha=1,2$. We denote by $\Gamma^{\alpha}$ the boundary of $\Omega^{\alpha}$, assumed to be "sufficiently" smooth. We assume that $\Gamma^{\alpha}=\Gamma_{D}^{\alpha} \cup \Gamma_{N}^{\alpha} \cup \Gamma_{c}^{\alpha}$ where $\left\{\Gamma_{D}^{\alpha}, \Gamma_{N}^{\alpha}, \Gamma_{c}^{\alpha}\right\}$ is a partition of $\Gamma^{\alpha}$ with mes $\left(\Gamma_{D}^{\alpha}\right)>0$ and $\operatorname{mes}\left(\Gamma_{c}^{\alpha}\right)>0$. On $\Gamma_{D}^{\alpha}$ a displacement is prescribed while on $\Gamma_{N}^{\alpha}$ a surface traction is prescribed. $\Gamma_{c}^{\alpha}$ is a part of $\Gamma^{\alpha}$ where both bodies may come in contact in the deformed configuration. 


\subsection{Equilibrium equations}

Let $\boldsymbol{u}^{\alpha}$ be the displacement fields of the body $\Omega^{\alpha}\left(\boldsymbol{u}^{\alpha}(x) \in \mathbb{R}^{d}\right)$. We set $\mathbf{u}=\left(\boldsymbol{u}^{1}, \boldsymbol{u}^{2}\right)$ the displacement field of the two-body system. Hooke's law is assumed for each elastic body, i.e. the stress tensor $\sigma^{\alpha}\left(\boldsymbol{v}^{\alpha}\right) \in\left(L^{2}\left(\Omega_{i}\right)\right)^{d \times d}$ relies on the strain tensor

$$
\epsilon\left(\boldsymbol{v}^{\alpha}\right)=\frac{1}{2}\left(\nabla \boldsymbol{v}^{\alpha}+\left(\nabla \boldsymbol{v}^{\alpha}\right)^{t}\right) \in\left(L^{2}\left(\Omega_{i}\right)\right)^{d \times d}
$$

through the linear equation

$$
\sigma^{\alpha}\left(\boldsymbol{v}^{\alpha}\right)=2 \mu_{\alpha} \epsilon\left(\boldsymbol{v}^{\alpha}\right)+\lambda_{\alpha} \operatorname{tr}\left(\epsilon\left(\boldsymbol{v}^{\alpha}\right)\right) \mathbb{I}_{d}
$$

where $\lambda_{\alpha} \geq 0$ and $\mu_{\alpha} \geq 0$ denote the Lamé constants and $\mathbb{I}_{d}$ the $d \times d$ identity matrix.

The equilibrium equations are

$$
\begin{aligned}
-\operatorname{div} \sigma^{i}\left(\boldsymbol{u}^{\alpha}\right) & =\boldsymbol{f}^{\alpha} \text { in } \Omega^{\alpha} \\
\sigma^{i}\left(\boldsymbol{u}^{\alpha}\right) \cdot \boldsymbol{n}^{\alpha} & =\boldsymbol{g}^{\alpha} \text { on } \Gamma_{N}^{\alpha}, \\
\boldsymbol{u}^{\alpha} & =0 \text { on } \Gamma_{D}^{\alpha},
\end{aligned}
$$

where $\boldsymbol{n}^{\alpha}$ stands for the unit outward normal to $\Omega^{\alpha}$.

\subsection{Contact and friction conditions}

For a complete formulation it remains to introduce the set of admissible displacement fields and the friction conditions. Let us denote by a subscript $H$ horizontal components and coordinates, e.g.

$$
\boldsymbol{x}_{H}=\left(x_{1}, x_{2}\right), \quad \boldsymbol{u}_{H}^{\alpha}=\left(u_{1}^{\alpha}, u_{2}^{\alpha}\right), \text { etc. }
$$

for $\boldsymbol{x}=\left(x_{1}, x_{2}, x_{3}\right)$ and $\boldsymbol{u}^{\alpha}=\left(u_{1}^{\alpha}, u_{2}^{\alpha}, u_{3}^{\alpha}\right)$. Following [19], we assume that $\Gamma_{c}^{1}$ lies above $\Gamma_{c}^{2}$ in the reference (undeformed) configuration, and that the contact surfaces are defined parametrically by

$$
\begin{array}{ll}
x_{3}=\psi^{1}\left(\boldsymbol{x}_{H}\right), & \left(\boldsymbol{x}_{H}, x_{3}\right) \in \Gamma_{c}^{1}, \\
x_{3}=\psi^{2}\left(\boldsymbol{x}_{H}\right), & \left(\boldsymbol{x}_{H}, x_{3}\right) \in \Gamma_{c}^{2} .
\end{array}
$$

The function $\psi^{\alpha}$ are assumed to be smooth. Let $\boldsymbol{x}_{H}^{1}$ be the coordinate label of a particle on the contact surface $\Gamma_{c}^{1}$ and $\boldsymbol{x}_{H}^{2}$ the unique particle in $\Gamma_{c}^{2}$ which is the closest to $\boldsymbol{x}_{H}^{1}$ in the (current) deformed configuration. Then a displacement field $\mathbf{u}=\left(\boldsymbol{u}^{1}, \boldsymbol{u}^{2}\right)$ is kinematically admissible if

$$
\begin{aligned}
\boldsymbol{x}_{H}^{1}+u_{H}^{1}\left(\boldsymbol{x}_{H}^{1}, \psi^{1}\left(\boldsymbol{x}_{H}^{1}\right)\right) & =\boldsymbol{x}_{H}^{2}+u_{H}^{2}\left(\boldsymbol{x}_{H}^{2}, \psi^{2}\left(\boldsymbol{x}_{H}^{2}\right)\right), \\
\psi^{1}\left(\boldsymbol{x}_{H}^{1}\right)+u_{3}^{1}\left(\boldsymbol{x}_{H}^{1}, \psi^{1}\left(\boldsymbol{x}_{H}^{1}\right)\right) & \geq \psi^{2}\left(\boldsymbol{x}_{H}^{2}\right)+u_{3}^{2}\left(\boldsymbol{x}_{H}^{2}, \psi^{2}\left(\boldsymbol{x}_{H}^{2}\right)\right) .
\end{aligned}
$$


To simplify the presentation, we sometimes write $\boldsymbol{u}^{\alpha}\left(\boldsymbol{x}_{H}^{\alpha}\right)$ instead of $\boldsymbol{u}^{\alpha}\left(\boldsymbol{x}_{H}^{\alpha}, \psi^{\alpha}\left(\boldsymbol{x}_{H}^{\alpha}\right)\right)$. Assuming infinitesimal deformations we have

$$
\begin{aligned}
\boldsymbol{u}_{H}^{2}\left(\boldsymbol{x}_{H}^{2}\right)=\boldsymbol{u}_{H}^{2}\left(\boldsymbol{x}_{H}^{2}, \psi^{2}\left(\boldsymbol{x}_{H}^{2}\right)\right) & =\boldsymbol{u}_{H}^{2}\left(\boldsymbol{x}_{H}^{1}+\boldsymbol{x}_{H}^{2}-\boldsymbol{x}_{H}^{1}, \psi^{1}\left(\boldsymbol{x}_{H}^{1}\right)+\psi^{2}\left(\boldsymbol{x}_{H}^{2}\right)-\psi^{1}\left(\boldsymbol{x}_{H}^{1}\right)\right) \\
& \approx \boldsymbol{u}_{H}^{2}\left(\boldsymbol{x}_{H}^{1}, \psi^{1}\left(\boldsymbol{x}_{H}^{1}\right)\right)
\end{aligned}
$$

by neglecting first order derivative terms, and

$\psi^{2}\left(\boldsymbol{x}_{H}^{2}\right) \approx \psi^{2}\left(\boldsymbol{x}_{H}^{1}\right)+\nabla \psi^{2}\left(\boldsymbol{x}_{H}^{1}\right) \cdot\left(\boldsymbol{x}_{H}^{2}-\boldsymbol{x}_{H}^{1}\right)=\psi^{2}\left(\boldsymbol{x}_{H}^{1}\right)+\nabla \psi^{2}\left(\boldsymbol{x}_{H}^{1}\right) \cdot\left(\boldsymbol{u}_{H}^{1}\left(\boldsymbol{x}_{H}^{1}\right)-\boldsymbol{u}_{H}^{2}\left(\boldsymbol{x}_{H}^{1}\right)\right)$

using (2.4). Plugging (2.6) and (2.7) into (2.5), we get

$$
\nabla \psi^{2}\left(\boldsymbol{x}_{H}^{1}\right) \cdot\left(\boldsymbol{u}_{H}^{1}-\boldsymbol{u}_{H}^{2}\right)-\left(u_{2}^{1}\left(\boldsymbol{x}_{H}^{1}\right)-u_{2}^{2}\left(\boldsymbol{x}_{H}^{1}\right)\right) \leq \psi^{1}\left(\boldsymbol{x}_{H}^{1}\right)-\psi^{2}\left(\boldsymbol{x}_{H}^{1}\right) .
$$

Setting

$$
\begin{aligned}
\boldsymbol{n} & =\left(\nabla \psi^{2}\left(\boldsymbol{x}_{H}^{1}\right),-1\right) / \sqrt{\left|\nabla \psi^{2}\left(\boldsymbol{x}_{H}^{1}\right)\right|^{2}+1} \\
g\left(\boldsymbol{x}_{H}^{1}\right) & =\left(\psi^{1}\left(\boldsymbol{x}_{H}^{1}\right)-\psi^{2}\left(\boldsymbol{x}_{H}^{1}\right)\right) / \sqrt{\left|\nabla \psi^{2}\left(\boldsymbol{x}_{H}^{1}\right)\right|^{2}+1} \\
{\left[\mathbf{u}_{n}\right] } & =\left(\boldsymbol{u}^{1}\left(\boldsymbol{x}_{H}^{1}\right)-\boldsymbol{u}^{2}\left(\boldsymbol{x}_{H}^{1}\right)\right) \cdot \boldsymbol{n},
\end{aligned}
$$

we obtain the contact condition

$$
\left[\mathbf{u}_{n}\right]-g \leq 0 \quad \text { on } \Gamma_{c}^{1} \text { and } \Gamma_{c}^{2} .
$$

The vector $\boldsymbol{n}$ stands for the unit outward normal to the boundary $\Gamma_{c}^{1}$ while $g$ is the normalized initial "gap" between $\Omega^{1}$ and $\Omega^{2}$. $\left[\mathbf{u}_{n}\right]$ is the relative normal displacement on the contact surface.

We can identify both surfaces $\Gamma_{c}^{\alpha}$ by their projection $\Gamma_{c}$ on the $\boldsymbol{x}_{H}$ plane so that the contact condition (2.8) can be replaced by

$$
\left[\mathbf{u}_{n}\right]-g \leq 0 \quad \text { on } \Gamma_{c}
$$

We define the relative tangential displacement on the contact surface $\Gamma_{c}$ by

$$
\left[\mathbf{u}_{t}\right]=\boldsymbol{u}_{t}^{1}-\boldsymbol{u}_{t}^{2}=\boldsymbol{u}^{1}-\boldsymbol{u}^{2}-\left[\mathbf{u}_{n}\right] \boldsymbol{n}=[\mathbf{u}]-\left[\mathbf{u}_{n}\right] \boldsymbol{n},
$$

where $[\mathbf{u}]=\left.\left(\boldsymbol{u}^{1}-\boldsymbol{u}^{2}\right)\right|_{\Gamma_{c}}$. Let $\boldsymbol{\sigma}_{n}$ and $\boldsymbol{\sigma}_{t}$ denote normal and tangential stress on $\Gamma_{c}$

$$
\begin{aligned}
\boldsymbol{\sigma}_{n} & =\left(\sigma^{1}\left(\boldsymbol{u}^{1}\right) \boldsymbol{n}\right) \cdot \boldsymbol{n}=-\left(\sigma^{2}\left(\boldsymbol{u}^{2}\right) \boldsymbol{n}\right) \cdot \boldsymbol{n} \\
\boldsymbol{\sigma}_{t} & =\sigma^{\alpha}\left(\boldsymbol{u}^{\alpha}\right) \boldsymbol{n}-\boldsymbol{\sigma}_{n} \boldsymbol{n} .
\end{aligned}
$$

The contact (nonpenetration) conditions on $\Gamma_{c}$ are

$$
\left[\mathbf{u}_{n}\right]-g \leq 0, \quad \boldsymbol{\sigma}_{n} \leq 0, \quad\left(\left[\mathbf{u}_{n}\right]-g\right) \boldsymbol{\sigma}_{n}=0, \text { on } \Gamma_{c} .
$$


In addition with the nonpenetration conditions (2.10), the following Tresca friction conditions are prescribed on $\Gamma_{c}$

$$
\begin{array}{ll}
s=\nu_{f}\left|\boldsymbol{\sigma}_{n}\right|, \quad\left|\boldsymbol{\sigma}_{t}\right|<s \Longrightarrow\left[\mathbf{u}_{t}\right]=0 \text { on } \Gamma_{c}, \\
\left|\boldsymbol{\sigma}_{t}\right|=s \Longrightarrow \exists \lambda \geq 0,\left[\mathbf{u}_{t}\right]=-\lambda \boldsymbol{\sigma}_{t} \text { on } \Gamma_{c},
\end{array}
$$

where $\nu_{f}$ stands for the (positive) friction coefficient and $s$ is considered as given.

\subsection{Constrained minimization problem}

Let us introduce Hilbert spaces of virtual displacements

$$
V^{\alpha}=\left\{\boldsymbol{v} \in\left(H^{1}\left(\Omega^{\alpha}\right)\right)^{d}: \boldsymbol{v}=0 \text { on } \Gamma_{D}^{\alpha}\right\}, \quad \alpha=1,2,
$$

and we set $\mathbf{V}=V^{1} \times V^{2}$. For $\boldsymbol{u}^{\alpha}, \boldsymbol{v}^{\alpha} \in V^{\alpha}$, we define the bilinear form of virtual works produced by the displacement $\boldsymbol{u}^{\alpha}$ by

$$
a^{\alpha}\left(\boldsymbol{u}^{\alpha}, \boldsymbol{v}^{\alpha}\right)=\int_{\Omega^{\alpha}} \sigma^{\alpha}\left(\boldsymbol{u}^{\alpha}\right) \epsilon\left(\boldsymbol{v}^{\alpha}\right) d x
$$

and the linear form of virtual works due to volume forces and surface traction by

$$
\ell^{\alpha}\left(\boldsymbol{v}^{\alpha}\right)=\int_{\Omega^{\alpha}} \boldsymbol{f}^{\alpha} \boldsymbol{v}^{\alpha} d x+\int_{\Gamma_{N}^{\alpha}} \boldsymbol{g}^{\alpha} \boldsymbol{v}^{\alpha} d \Gamma .
$$

For each body, we define the total potential energy functional $J^{\alpha}$ by

$$
J^{\alpha}\left(\boldsymbol{v}^{\alpha}\right)=\frac{1}{2} a^{\alpha}\left(\boldsymbol{v}^{\alpha}, \boldsymbol{v}^{\alpha}\right)-\ell^{\alpha}\left(\boldsymbol{v}^{\alpha}\right), \quad \forall \boldsymbol{v}^{\alpha} \in V^{\alpha}
$$

and we set

$$
\boldsymbol{J}(\mathbf{v})=\sum_{\alpha=1}^{2} J^{\alpha}\left(\boldsymbol{v}^{\alpha}\right), \quad \mathbf{v} \in \mathbf{V},
$$

the total potential energy of the two-body system. Assuming that $\operatorname{mes}\left(\Gamma_{D}^{\alpha}\right)>0$, the functional $\boldsymbol{J}$ is convex, G-differentiable and coercive on $\mathbf{V}$.

We now introduce the set of kinematically admissible displacement fields

$$
K=\left\{\mathbf{v}=\left(\boldsymbol{v}^{1}, \boldsymbol{v}^{2}\right) \in \mathbf{V},\left[\boldsymbol{v}_{n}\right]-g \leq 0 \text { on } \Gamma_{c}\right\}
$$

and the friction functional

$$
j(\mathbf{v})=\int_{\Gamma_{c}} s\left|\boldsymbol{v}_{t}^{1}-\boldsymbol{v}_{t}^{2}\right| d \Gamma=\int_{\Gamma_{c}} s\left|\left[\mathbf{v}_{t}\right]\right| d \Gamma,
$$

where $|\cdot|$ denotes the Euclidean norm. The functional $j$ is continuous and convex but nondifferentiable. 
With the above preparations, the unilateral contact problem with Tresca friction can be formulated as the constrained minimization problem

Find $\mathbf{u} \in K$ such that

$$
\boldsymbol{J}(\mathbf{u})+j(\mathbf{u}) \leq \boldsymbol{J}(\mathbf{v})+j(\mathbf{v}), \quad \forall \mathbf{v} \in K .
$$

With the assumption $\operatorname{mes}\left(\Gamma_{D}^{\alpha}\right)>0$, the functional $\boldsymbol{J}+j$ is strictly convex and coercive, then there exists a unique solution to (2.15).

\section{Domain decomposition}

In this section we present our domain decomposition method for solving (2.15). To this end, we need to transform the convex minimization problem (2.15) into a suitable saddle-point problem by introducing auxiliary interface unknowns.

\subsection{Augmented Lagrangian formulation}

Let us introduce auxiliary unknowns $\boldsymbol{q}_{c}=\left(q_{c}^{1}, q_{c}^{2}\right)$ and $\boldsymbol{q}_{f}=\left(q_{f}^{1}, q_{f}^{2}\right)$. Following Fortin and Glowinski [12] or Glowinski and Le Tallec [13], we introduce the set

$$
C=\left\{\boldsymbol{q}_{c}=\left(q_{c}^{1}, q_{c}^{2}\right) \in\left(L^{2}\left(\Gamma_{c}\right)\right)^{2}, q_{c}^{1}-q_{c}^{2}-g \leq 0 \text { on } \Gamma_{c}\right\}
$$

and its characteristic functional $I_{C}:\left(L^{2}\left(\Gamma_{c}\right)\right)^{2} \rightarrow \mathbb{R} \cup\{+\infty\}$ defined by

$$
I_{C}\left(\boldsymbol{q}_{c}\right)= \begin{cases}0 & \text { if } \boldsymbol{q}_{c} \in C \\ +\infty & \text { if } \boldsymbol{q}_{c} \notin C\end{cases}
$$

Setting

$$
\mathbf{q}=\left(\boldsymbol{q}_{c}, \boldsymbol{q}_{f}\right), \quad H_{c}=\left(L^{2}\left(\Gamma_{c}\right)\right)^{2}, \quad H_{f}=\left(L^{2}\left(\Gamma_{c}\right)\right)^{2(d-1)} \quad \text { and } H=H_{c} \times H_{f},
$$

it is clear that (2.15) is equivalent to the following constrained minimization problem

Find $(\mathbf{u}, \mathbf{p}) \in \mathbf{V} \times H$ such that

$$
\begin{aligned}
\boldsymbol{J}(\mathbf{u})+j\left(\boldsymbol{p}_{f}\right)+I_{C}\left(\boldsymbol{p}_{c}\right) & \leq J(\mathbf{v})+j\left(\boldsymbol{q}_{f}\right)+I_{C}\left(\boldsymbol{q}_{c}\right) \quad \forall(\mathbf{v}, \mathbf{q}) \in \mathbf{V} \times H, \\
\boldsymbol{u}_{n}^{\alpha}-p_{c}^{\alpha} & =0 \text { on } \Gamma_{c}, \alpha=1,2, \\
\boldsymbol{u}_{t}^{\alpha}-p_{f}^{\alpha} & =0 \text { on } \Gamma_{c}, \alpha=1,2 .
\end{aligned}
$$

To equations (3.1)-(3.3) we associate the augmented Lagrangian functional $\mathscr{L}_{r}$ defined on $\mathbf{V} \times H \times H$ by

$$
\begin{aligned}
\mathscr{L}_{r}(\mathbf{v}, \mathbf{q} ; \boldsymbol{\mu}) & =\boldsymbol{J}(\boldsymbol{v})+j\left(\boldsymbol{q}_{f}\right)+I_{C}\left(\boldsymbol{q}_{c}\right)+\sum_{\alpha=1}^{2}\left[\left(\mu_{c}^{\alpha}, \boldsymbol{v}_{n}^{\alpha}-q_{c}^{\alpha}\right)_{\Gamma_{c}}+\left(\mu_{f}^{\alpha}, \boldsymbol{v}_{t}^{\alpha}-q_{f}^{\alpha}\right)_{\Gamma_{c}}\right] \\
& +\frac{r}{2} \sum_{\alpha=1}^{2}\left(\left\|\boldsymbol{v}_{n}^{\alpha}-q_{c}^{\alpha}\right\|_{0, \Gamma_{c}}^{2}+\left\|\boldsymbol{v}_{t}^{\alpha}-q_{f}^{\alpha}\right\|_{0, \Gamma_{c}}^{2}\right),
\end{aligned}
$$


where $r>0$ is the penalty parameter and $\boldsymbol{\mu}=\left(\boldsymbol{\mu}_{c}, \boldsymbol{\mu}_{f}\right)$. The corresponding saddle-point problem is

Find $((\mathbf{u}, \mathbf{p}), \boldsymbol{\lambda}) \in \mathbf{V} \times H \times H$ such that

$$
\mathscr{L}_{r}(\mathbf{u}, \mathbf{p} ; \boldsymbol{\mu}) \leq \mathscr{L}_{r}(\mathbf{v}, \mathbf{q} ; \boldsymbol{\mu}) \leq \mathscr{L}_{r}(\mathbf{v}, \mathbf{q} ; \boldsymbol{\lambda}), \quad \forall((\mathbf{v}, \mathbf{q}), \boldsymbol{\mu}) \in \mathbf{V} \times H \times H,
$$

where we have set $\boldsymbol{\lambda}=\left(\boldsymbol{\lambda}_{c}, \boldsymbol{\lambda}_{f}\right)$.

\subsection{Uzawa block relaxation method}

A saddle-point of $\mathscr{L}_{r}$ can be determined by a standard Uzawa method for augmented Lagrangian, see e.g. $[3,4]$. The main difficulty with the standard Uzawa method is the coupling of subdomains and unknowns $\boldsymbol{u}$ and p. By introducing auxiliary unknowns, $\boldsymbol{p}_{c}$ and $\boldsymbol{p}_{f}$, we have implicitly split the problem in a "linear part" (subproblem in $\mathbf{u}$ ) and a "nonlinear part" (subproblem in p). Furthermore, the displacement fields on subdomains $\Omega^{1}$ and $\Omega^{2}$ are now linked only through the auxiliary unknowns. To take advantage of these properties, a quite natural method consists of using a Uzawa block relaxation method.

Uzawa block relaxation methods have been used in nonlinear mechanics for operator splitting and domain decomposition methods [12, 13, 21]. Applying a Uzawa block relaxation (UBR) method to the saddle-point problem (3.5), we obtain the following algorithm.

\section{Algorithm UBR}

Initialization. $\mathbf{p}^{-1}=\left(\boldsymbol{p}_{c}^{-1}, \boldsymbol{p}_{f}^{-1}\right)$ and $\boldsymbol{\lambda}^{0}=\left(\boldsymbol{\lambda}_{c}^{0}, \boldsymbol{\lambda}_{f}^{0}\right)$ are given.

Iteration $k \geq 0$. Compute successively $\mathbf{u}^{k}=\left(\boldsymbol{u}^{1, k}, \boldsymbol{u}^{2, k}\right), \mathbf{p}^{k}=\left(\boldsymbol{p}_{c}^{k}, \boldsymbol{p}_{f}^{k}\right)$ and $\boldsymbol{\lambda}^{k+1}=$ $\left(\boldsymbol{\lambda}_{c}^{k+1}, \boldsymbol{\lambda}_{f}^{k+1}\right)$ as follows.

- Find $\mathbf{u}^{k} \in \mathbf{V}$ such that

$$
\mathscr{L}_{r}\left(\mathbf{u}^{k}, \mathbf{p}^{k-1} ; \boldsymbol{\lambda}^{k}\right) \leq \mathscr{L}_{r}\left(\mathbf{v}, \mathbf{p}^{k-1} ; \boldsymbol{\lambda}^{k}\right), \quad \forall \mathbf{v} \in \mathbf{V}
$$

- Find $\mathbf{p}^{k}=\left(\boldsymbol{p}_{c}^{k}, \boldsymbol{p}_{f}^{k}\right) \in H$ such that

$$
\mathscr{L}_{r}\left(\mathbf{u}^{k}, \mathbf{p}^{k} ; \boldsymbol{\lambda}^{k}\right) \leq \mathscr{L}_{r}\left(\mathbf{u}^{k}, \mathbf{q} ; \boldsymbol{\lambda}^{k}\right), \quad \forall \mathbf{q} \in H .
$$

- Update the Lagrange multipliers

$$
\begin{aligned}
& \lambda_{c}^{\alpha, k+1}=\lambda_{c}^{\alpha, k}+r\left(\boldsymbol{u}_{n}^{\alpha, k}-p_{c}^{\alpha, k}\right), \alpha=1,2, \\
& \lambda_{f}^{\alpha, k+1}=\lambda_{f}^{\alpha, k}+r\left(\boldsymbol{u}_{t}^{\alpha, k}-p_{f}^{\alpha, k}\right), \alpha=1,2 .
\end{aligned}
$$

We detail the above algorithm in the next subsections. 


\subsection{Solution of subproblem (3.6)}

Since the functional $\mathbf{v} \mapsto \mathscr{L}_{r}\left(\mathbf{v}, \mathbf{p}^{k-1}, \boldsymbol{\lambda}^{k}\right)$ is convex and Gâteau-differentiable on $\mathbf{V}$, the solution of (3.6) can be characterized by the Euler-Lagrange equation

$$
\frac{\partial}{\partial \mathbf{v}} \mathscr{L}_{r}\left(\mathbf{u}^{k}, \mathbf{p}_{c}^{k-1}, \boldsymbol{\lambda}_{c}^{k}\right) \cdot \mathbf{v}=0, \quad \forall \mathbf{v} \in \mathbf{V}
$$

A straightforward calculation yields

Find $\boldsymbol{u}^{\alpha, k} \in V^{\alpha}$ such that

$$
\begin{aligned}
a^{\alpha}\left(\boldsymbol{u}^{\alpha, k}, \boldsymbol{v}^{\alpha}\right)+r\left(\boldsymbol{u}_{n}^{\alpha, k}, \boldsymbol{v}_{n}^{\alpha}\right)_{\Gamma_{c}} & +r\left(\boldsymbol{u}_{t}^{\alpha, k}, \boldsymbol{v}_{t}^{\alpha}\right)_{\Gamma_{c}}=\ell^{\alpha}\left(\boldsymbol{v}^{\alpha}\right)+\left(r p_{c}^{\alpha, k-1}-\lambda_{c}^{\alpha, k}, \boldsymbol{v}_{n}^{\alpha}\right)_{\Gamma_{c}} \\
& +\left(r p_{f}^{\alpha, k-1}-\lambda_{f}^{\alpha, k}, \boldsymbol{v}_{t}^{\alpha}\right)_{\Gamma_{c}} \quad \forall \boldsymbol{v}^{\alpha} \in V^{\alpha}, \alpha=1,2 .
\end{aligned}
$$

The main property of the Uzawa block relaxation now appears: the linear elasticity problems on each subdomain are now uncoupled.

\subsection{Solution of subproblem (3.7)}

Note that in (3.7) subproblems in $\boldsymbol{p}_{c}$ and $\boldsymbol{p}_{f}$ are uncoupled. Consequently, we can minimize the functional $\mathbf{q}=\left(\boldsymbol{q}_{c}, \boldsymbol{q}_{f}\right) \mapsto \mathscr{L}_{r}\left(\mathbf{u}^{k}, \mathbf{q}, \boldsymbol{\lambda}^{k}\right)$ separately in $\boldsymbol{q}_{c}$ and $\boldsymbol{q}_{f}$.

\subsubsection{Nonpenetration subproblem}

Over the nonpenetration constraints set $C$ the functional $\boldsymbol{q}_{c} \mapsto \mathscr{L}_{r}\left(\mathbf{u}^{k}, \boldsymbol{q}_{c}, \boldsymbol{\lambda}^{k}\right)$ can be simplified

$$
\Phi_{c}\left(\boldsymbol{q}_{c}\right):=\mathscr{L}_{r}\left(\mathbf{u}^{k}, \boldsymbol{q}_{c}, \boldsymbol{q}_{f}, \boldsymbol{\lambda}^{k}\right)=\sum_{\alpha=1}^{2}\left[\frac{r}{2}\left\|q_{c}^{\alpha}\right\|_{0, \Gamma_{c}}^{2}-\left(\lambda_{c}^{\alpha, k}+r \boldsymbol{u}_{n}^{\alpha, k}, p_{c}^{\alpha}\right)_{\Gamma_{c}}\right]+\beta,
$$

where $\beta$ is a constant which does not count in the minimization. The functional $\Phi_{c}$ is convex and coercive over the convex set $C$. The infimum $\boldsymbol{p}_{c}^{k}$ of the functional $\Phi_{c}$ must satisfied the Kuhn-Tucker conditions

$$
\begin{aligned}
\sum_{\alpha=1}^{2}\left[r\left(p_{c}^{\alpha, k}, q_{c}^{\alpha}\right)_{\Gamma_{c}}-\left(\lambda_{c}^{\alpha, k}+r \boldsymbol{u}_{n}^{\alpha, k}, q_{c}^{\alpha}\right)_{\Gamma_{c}}\right]+\left(\gamma^{k}, q_{c}^{1}-q_{c}^{2}\right)_{\Gamma_{c}} & =0, \quad \forall\left(q_{c}^{1}, q_{c}^{2}\right), \\
\left(\gamma^{k}, p_{c}^{1, k}-p_{c}^{2, k}-g\right)_{\Gamma_{c}} & =0
\end{aligned}
$$

where $\gamma^{k} \geq 0$ is referred as a Lagrange (Kuhn-Tucker) multiplier for the constraints set $C$ (see e.g. [17, 25]). From (3.9) we deduce that

$$
p_{c}^{\alpha, k}=\boldsymbol{u}_{n}^{\alpha, k}+\frac{1}{r}\left(\lambda_{c}^{\alpha, k}+(-1)^{\alpha} \gamma^{k}\right), \quad \alpha=1,2 .
$$

Substituting (3.11) into (3.10), we obtain

$$
\left(\gamma^{k}, \frac{1}{r}\left(\left[\boldsymbol{\lambda}^{k}\right]+r\left[\mathbf{u}_{n}^{k}\right]-2 \gamma^{k}\right)-g\right)_{\Gamma_{c}}=0,
$$


where we have set $\left[\boldsymbol{\lambda}_{c}^{k}\right]=\lambda_{c}^{1, k}-\lambda_{c}^{2, k}$. From (3.10) if $\gamma^{k}>0$, we must have

$$
\frac{1}{r}\left(\left[\boldsymbol{\lambda}_{c}^{k}\right]+r\left[\mathbf{u}_{n}^{k}\right]-2 \gamma^{k}\right)-g=0
$$

implying that

$$
\gamma^{k}=\frac{1}{2}\left(\left[\boldsymbol{\lambda}_{c}^{k}\right]+r\left(\left[\mathbf{u}_{n}^{k}\right]-g\right)\right)
$$

Since $\gamma^{k} \geq 0$, we set

$$
\gamma^{k}=\frac{1}{2}\left(\left[\boldsymbol{\lambda}_{c}^{k}\right]+r\left(\left[\mathbf{u}_{n}^{k}\right]-g\right)\right)^{+}:=\max \left\{0, \frac{1}{2}\left(\left[\boldsymbol{\lambda}_{c}^{k}\right]+r\left(\left[\mathbf{u}_{n}^{k}\right]-g\right)\right)\right\} .
$$

Substituting (3.12) into (3.11), we deduce the auxiliary contact unknowns

$$
p_{c}^{\alpha, k}=\boldsymbol{u}_{n}^{\alpha, k}+\frac{1}{r} \lambda_{c}^{\alpha, k}+\frac{(-1)^{\alpha}}{2 r}\left(\left[\boldsymbol{\lambda}_{c}^{k}\right]+r\left(\left[\mathbf{u}_{n}^{k}\right]-g\right)\right)^{+}, \quad \alpha=1,2 .
$$

We easily verify that

- if $\gamma^{k}>0$, then $p_{c}^{1, k}-p_{c}^{2, k}-g=0$;

- if $\gamma^{k}=0$, then $p_{c}^{1, k}-p_{c}^{2, k}-g \leq 0$.

\subsubsection{Friction subproblem}

We now compute the solution of the Tresca friction subproblem. The functional $\boldsymbol{q}_{f} \mapsto$ $\mathscr{L}_{r}\left(\mathbf{u}^{k}, \boldsymbol{q}_{f}, \boldsymbol{\lambda}^{k}\right)$ can be rewritten as

$$
\Phi_{f}\left(\boldsymbol{q}_{f}\right):=\frac{r}{2}\left\|\boldsymbol{q}_{f}\right\|_{0, \Gamma_{c}}^{2}-\left(\mathbf{b}^{k}, \boldsymbol{q}_{f}\right)_{\Gamma_{c}}+\int_{\Gamma_{c}} s\left|q_{f}^{1}-q_{f}^{2}\right| d \Gamma+\beta,
$$

where, for clarity, we have set

$$
b^{\alpha, k}=\lambda_{f}^{\alpha, k}+r \boldsymbol{u}_{t}^{\alpha, k}, \quad \alpha=1,2, \quad \mathbf{b}^{k}=\left(b^{1, k}, b^{2, k}\right)
$$

and $\beta$ a constant which does not count in the minimization. The friction subproblem is therefore

Find $\boldsymbol{p}_{f} \in H_{f}$ such that

$$
\Phi_{f}\left(\boldsymbol{p}_{f}\right) \leq \Phi_{f}\left(\boldsymbol{q}_{f}\right), \quad \forall \boldsymbol{q}_{f} \in H_{f} .
$$

Let $\mathcal{F}: X=H_{f} \rightarrow \mathbb{R}$ and $\mathcal{G}: Y=\left(L^{2}\left(\Gamma_{c}\right)\right)^{d-1} \rightarrow \mathbb{R}$ be defined by

$$
\begin{aligned}
\mathcal{F}\left(\boldsymbol{q}_{f}\right) & =\frac{r}{2}\left\|\boldsymbol{q}_{f}\right\|_{0, \Gamma_{c}}^{2}-\left(\mathbf{b}^{k}, \boldsymbol{q}_{f}\right)_{\Gamma_{c}}=\sum_{\alpha=1}^{2}\left(\frac{r}{2}\left\|q_{f}^{\alpha}\right\|_{0, \Gamma_{c}}+\left(b^{\alpha, k}, q_{f}^{\alpha}\right)_{\Gamma_{c}}\right), \\
\mathcal{G}(y) & =\int_{\Gamma_{c}} s|y| d \Gamma .
\end{aligned}
$$


The functionals $\mathcal{F}$ and $\mathcal{G}$ are convex and continuous. We introduce the bounded linear operator $\Lambda \in \mathcal{L}(X, Y)$ defined by

$$
\Lambda \boldsymbol{q}_{f}=q_{f}^{1}-q_{f}^{2}=\left[\boldsymbol{q}_{f}\right]
$$

so that,

$$
\mathcal{G}\left(\Lambda \boldsymbol{q}_{f}\right)=\int_{\Gamma_{c}} s\left|\left[\boldsymbol{q}_{f}\right]\right| d \Gamma
$$

The minimization problem (3.15) becomes

$$
(P) \quad \inf _{\boldsymbol{q}_{f} \in X} \mathcal{F}\left(\boldsymbol{q}_{f}\right)+\mathcal{G}\left(\Lambda \boldsymbol{q}_{f}\right)
$$

The Fenchel dual problem of $(P)$ is

$$
\left(P^{*}\right) \sup _{y^{*} \in Y^{*}}-\mathcal{F}\left(-\Lambda^{*} y^{*}\right)-\mathcal{G}\left(y^{*}\right)
$$

where $\Lambda^{*} \in \mathcal{L}\left(Y^{*}, X^{*}\right)$ is the adjoint of $\Lambda$ and $\mathcal{F}^{*}: X^{*}=X \rightarrow \mathbb{R} \cup\{\infty\}, \mathcal{G}^{*}: Y^{*}=$ $Y \rightarrow \mathbb{R} \cup\{\infty\}$ denote the Fenchel convex conjugate functionals (see e.g. [10]) of $\mathcal{F}$ and $\mathcal{G}$, respectively. It is easy to see that $\mathcal{F}$ and $\mathcal{G}$ satisfy the conditions of the Fenchel duality theorem [10, p. 59] and thus it follows that no duality gap occurs. Then the solutions $\boldsymbol{p}_{f}$ and $\bar{y}^{*}$ of $(P)$ and $\left(P^{*}\right)$ satisfy the extremality condition $[10$, p. 53]

$$
-\Lambda^{*} \bar{y}^{*} \in \partial \mathcal{F}\left(\boldsymbol{p}_{f}\right)
$$

where $\partial$ denotes the subdifferential.

Let $\boldsymbol{q}_{f}^{*} \in X^{*}$ be given. From the definition of the Fenchel convex conjugate we have

$$
\begin{aligned}
\mathcal{F}^{*}\left(\boldsymbol{q}_{f}^{*}\right) & =\sup _{\boldsymbol{q}_{f} \in X}\left(\boldsymbol{q}_{f}^{*}, \boldsymbol{q}_{f}\right)_{\Gamma_{c}}-\frac{r}{2}\left\|\boldsymbol{q}_{f}\right\|_{0, \Gamma_{c}}^{2}+\left(\mathbf{b}^{k}, \boldsymbol{q}_{f}\right)_{\Gamma_{c}} \\
& =\sup _{\boldsymbol{q}_{f} \in X}\left(\boldsymbol{q}_{f}^{*}+\mathbf{b}^{k}, \boldsymbol{q}_{f}\right)_{\Gamma_{c}}-\frac{r}{2}\left\|\boldsymbol{q}_{f}\right\|_{0, \Gamma_{c}}^{2} \\
& =\frac{1}{2 r}\left\|\boldsymbol{q}_{f}^{*}+\mathbf{b}^{k}\right\|_{0, \Gamma_{c}}^{2}=\frac{1}{2 r} \sum_{\alpha=1}^{2}\left\|q_{f}^{\alpha, *}+b^{\alpha, k}\right\|_{0, \Gamma_{c}}^{2} .
\end{aligned}
$$

For $y^{*} \in Y^{*}$, the Fenchel convex conjugate functional of $\mathcal{G}$ is

$$
\mathcal{G}^{*}\left(y^{*}\right)=\sup _{y \in Y}\left(y^{*}, y\right)_{\Gamma_{c}}-\int_{\Gamma_{c}} s|y| d \Gamma= \begin{cases}0 & \text { if }\left|y^{*}\right| \leq s \\ +\infty & \text { if }\left|y^{*}\right| \geq s\end{cases}
$$

From (3.19), we have

$$
\mathcal{F}^{*}\left(-\Lambda^{*} y^{*}\right)=\mathcal{F}^{*}\left(-y^{*}, y^{*}\right)=\frac{1}{2 r}\left(\left\|b^{1, k}-y^{*}\right\|_{0, \Gamma_{c}}^{2}+\left\|b^{2, k}+y^{*}\right\|_{0, \Gamma_{c}}^{2}\right) .
$$


Then the dual problem $\left(P^{*}\right)$ becomes

$$
\sup _{y^{*} \in Y^{*}}-\mathcal{F}\left(-\Lambda^{*} y^{*}\right)-\mathcal{G}\left(y^{*}\right)=\sup _{\left|y^{*}\right| \leq s}-\frac{1}{2 r}\left(\left\|b^{1, k}-y^{*}\right\|_{0, \Gamma_{c}}^{2}+\left\|b^{2, k}+y^{*}\right\|_{0, \Gamma_{c}}^{2}\right) .
$$

The unique solution of this problem is

$$
\bar{y}^{*}= \begin{cases}\frac{1}{2}\left[\mathbf{b}^{k}\right] & \text { if }|[\mathbf{b}]| \leq 2 s, \\ s \frac{\left[\mathbf{b}^{k}\right]}{\left|\left[\mathbf{b}^{k}\right]\right|} & \text { if }|[\mathbf{b}]| \geq 2 s .\end{cases}
$$

To compute the primal solution $\boldsymbol{p}_{f}$ from the dual solution $\bar{y}^{*}$ given by $(3.21)$, we use (3.19) and the extremality condition (3.18) $\left(\mathcal{F}^{*}\right.$ being a differentiable functional)

$$
\nabla \mathcal{F}\left(\boldsymbol{p}_{f}\right)=-\Lambda^{*} \bar{y}^{*}=\left(\begin{array}{c}
-\bar{y}^{*} \\
\bar{y}^{*}
\end{array}\right)
$$

A straightforward calculation yields, for $\alpha=1,2$,

$$
\begin{aligned}
& p_{f}^{\alpha, k}=\frac{1}{2 r}\left(b^{1, k}+b^{2, k}\right), \quad \text { if }\left|\left[\mathbf{b}^{k}\right]\right| \leq 2 s, \\
& p_{f}^{\alpha, k}=\frac{1}{r} b^{\alpha, k}+\frac{(-1)^{\alpha}}{r} s \frac{\left[\mathbf{b}^{k}\right]}{\left|\left[\mathbf{b}^{k}\right]\right|}, \quad \text { if }\left|\left[\mathbf{b}^{k}\right]\right| \geq 2 s .
\end{aligned}
$$

Substituting (3.14) into (3.22)-(3.23), we obtain the solution of the Tresca friction subproblem

$p_{f}^{\alpha, k}=\left\{\begin{array}{ll}\frac{1}{2 r} \sum_{\alpha=1}^{2}\left(\lambda_{f}^{\alpha, k}+r \boldsymbol{u}_{t}^{\alpha, k}\right) & \text { if }\left|\left[\boldsymbol{\lambda}_{f}^{k}\right]+r\left[\mathbf{u}_{t}\right]\right| \leq 2 s, \\ \boldsymbol{u}_{t}^{\alpha, k}+\frac{1}{r} \lambda_{f}^{\alpha, k}+\frac{(-1)^{\alpha} s}{r} \frac{\left[\boldsymbol{\lambda}_{f}^{k}\right]+r\left[\mathbf{u}_{t}^{k}\right]}{\left|\left[\boldsymbol{\lambda}_{f}^{k}\right]+r\left[\mathbf{u}_{t}^{k}\right]\right|} & \text { if }\left|\left[\boldsymbol{\lambda}^{k}\right]+r\left[\mathbf{u}_{t}^{k}\right]\right| \geq 2 s\end{array} \quad, \quad \alpha=1,2\right.$.

\subsection{Domain decomposition algorithm}

With the results of the previous subsections, we can now present our Uzawa block relaxation domain decomposition algorithm.

\section{Algorithm UBR-DDM}

Initialization. $\mathbf{p}^{-1}=\left(\boldsymbol{p}_{c}^{-1}, \boldsymbol{p}_{f}^{-1}\right)$ and $\boldsymbol{\lambda}^{0}=\left(\boldsymbol{\lambda}_{c}^{0}, \boldsymbol{\lambda}_{f}^{0}\right)$ are given.

Iteration $k \geq 0$. Compute successively $\boldsymbol{u}^{k}, \mathbf{p}^{k}$ and $\boldsymbol{\lambda}^{k+1}$ as follows

- Find $\boldsymbol{u}^{\alpha, k} \in V^{\alpha}$ such that

$$
\begin{aligned}
a^{\alpha}\left(\boldsymbol{u}^{\alpha, k}, \boldsymbol{v}^{\alpha}\right)+r\left(\boldsymbol{u}_{n}^{\alpha, k}, \boldsymbol{v}_{n}^{\alpha}\right)_{\Gamma_{c}} & +r\left(\boldsymbol{u}_{t}^{\alpha, k}, \boldsymbol{v}_{t}^{\alpha}\right)_{\Gamma_{c}}=\ell^{\alpha}\left(\boldsymbol{v}^{\alpha}\right)+\left(r p_{c}^{\alpha, k-1}-\lambda_{c}^{\alpha, k}, \boldsymbol{v}_{n}^{\alpha}\right)_{\Gamma_{c}} \\
& +\left(r p_{f}^{\alpha, k-1}-\lambda_{f}^{\alpha, k}, \boldsymbol{v}_{t}^{\alpha}\right)_{\Gamma_{c}} \quad \forall \boldsymbol{v}^{\alpha} \in V^{\alpha}, \alpha=1,2 .
\end{aligned}
$$


- Compute the auxiliary interface unknowns $(\alpha=1,2)$

$$
\begin{aligned}
& p_{c}^{\alpha, k}=\boldsymbol{u}_{n}^{\alpha, k}+\frac{1}{r} \lambda_{c}^{\alpha, k}+\frac{(-1)^{\alpha}}{2 r}\left(\left[\boldsymbol{\lambda}_{c}^{k}\right]+r\left(\left[\mathbf{u}_{n}^{k}\right]-g\right)\right)^{+}, \\
& p_{f}^{\alpha, k}= \begin{cases}\frac{1}{2 r}\left(\lambda_{f}^{1, k}+\lambda_{f}^{2, k}+r\left(\boldsymbol{u}_{t}^{1, k}+\boldsymbol{u}_{t}^{2, k}\right)\right) & \text { if }\left|\left[\boldsymbol{\lambda}^{k}\right]+r\left[\mathbf{u}_{t}\right]\right| \leq 2 s, \\
\boldsymbol{u}_{t}^{\alpha, k}+\frac{1}{r} \lambda_{f}^{\alpha, k}+\frac{(-1)^{\alpha} s}{r} \frac{\left[\boldsymbol{\lambda}^{k}\right]+r\left[\mathbf{u}_{t}^{k}\right]}{\left|\left[\boldsymbol{\lambda}^{k}\right]+r\left[\mathbf{u}_{t}^{k}\right]\right|} & \text { if }\left|\left[\boldsymbol{\lambda}^{k}\right]+r\left[\mathbf{u}_{t}^{k}\right]\right| \geq 2 s\end{cases}
\end{aligned}
$$

- Update the Lagrange multipliers

$$
\begin{aligned}
& \lambda_{c}^{\alpha, k+1}=\lambda_{c}^{\alpha, k}+r\left(\boldsymbol{u}_{n}^{\alpha, k}-p_{c}^{\alpha, k}\right), \alpha=1,2, \\
& \lambda_{f}^{\alpha, k+1}=\lambda_{f}^{\alpha, k}+r\left(\boldsymbol{u}_{t}^{\alpha, k}-p_{f}^{\alpha, k}\right), \alpha=1,2 .
\end{aligned}
$$

We iterate until the relative error on $\boldsymbol{u}^{k}, \boldsymbol{p}_{c}^{k}$ and $\boldsymbol{p}_{f}^{k}$ becomes sufficiently small. The parallelizability of the above algorithm is obvious since, in every iteration, we solve in parallel two linear elasticity subproblems. Furthermore, the left-hand sides of the linear elasticity subproblems do not change during the iterative process implying the saving of computational cost due to matrix factorizations.

\section{Convergence results}

We first rewrite the constrained optimization problem (2.15) in a standard form. To this end we set

$$
G(\mathbf{v})=\boldsymbol{J}(\mathbf{v}), \quad F(\mathbf{q})=I_{C}\left(\boldsymbol{q}_{c}\right)+j\left(\boldsymbol{q}_{f}\right)
$$

and we introduce the linear and continuous operator $B$, from $\mathbf{V} \rightarrow L^{2}\left(\Gamma_{c}\right) \times L_{T}^{2}\left(\Gamma_{c}\right)$, defined by

$$
B \mathbf{v}=\left(\begin{array}{c}
{\left[\mathbf{v}_{n}\right]-g} \\
{\left[\mathbf{v}_{t}\right]}
\end{array}\right)
$$

where

$$
L_{T}^{2}\left(\Gamma_{c}\right)=\left\{\boldsymbol{v} \in\left(L^{2}\left(\Gamma_{c}\right)\right)^{d} \mid \boldsymbol{v}_{n}=0\right\} .
$$

We observe that (2.15) is equivalent to

Find $\mathbf{u} \in \mathbf{V}$ such that

$$
G(\mathbf{u})+F(B \mathbf{u}) \leq G(\mathbf{v})+F(B \mathbf{v}), \quad \forall \mathbf{v} \in \mathbf{V} .
$$

The functional $G$ is convex, proper and lower semi-continuous while $F$ is strictly convex and continuous. Furthermore, $G$ is uniformly convex on the bounded sets of $\mathbf{V}$. Algorithm UBR-DDM is therefore equivalent to the operator-splitting standard algorithm ALG2 described, e.g., in [13, ch. 3] or [12, ch. 3]. We have the following convergence theorem, see e.g. [13, ch. 3 , theorem 4.2]. 
Theorem 4.1 (Convergence). The sequence $\left(\mathbf{u}^{k}, \boldsymbol{p}^{k}, \boldsymbol{\lambda}^{k}\right)$ generated in Algorithm UBR$D D M$ is such that

$$
\mathbf{u}^{k} \rightarrow \mathbf{u} \text { in } \mathbf{V}, \quad \mathbf{p}^{k} \rightarrow \mathbf{p} \text { in } H, \quad \boldsymbol{\lambda}^{k} \rightarrow \boldsymbol{\lambda} \text { in } H
$$

$(\mathbf{u}, \mathbf{p}, \boldsymbol{\lambda})$ being a saddle-point of $\mathscr{L}_{r}$.

We now investigate how to recover the normal and tangential stress at the end of the the domain decomposition algorithm.

Corollary 4.2 (Normal stress). The contact pressure is given by $\boldsymbol{\sigma}_{n}=-\left[\boldsymbol{\lambda}_{c}\right] / 2$.

Proof. Since the Lagrange multiplier $\bar{\lambda}_{c}$ associated with the contact condition (2.9) is the negative stress in the normal direction, we deduce from (3.12) that $\gamma^{k} \rightarrow\left[\boldsymbol{\lambda}_{c}\right] / 2=\bar{\lambda}_{c}$.

Corollary 4.3 (Tangential stress). The friction stress is given by $\boldsymbol{\sigma}_{t}=-\left[\boldsymbol{\lambda}_{f}\right] / 2$.

Proof. As in the case of the contact condition, the Lagrange multiplier $\bar{\lambda}_{f}$, associated with the non-differentiability of the friction functional $j$, has a mechanical interpretation

$$
\bar{\lambda}_{f}=-\sigma_{t}
$$

Since $s \in L^{2}\left(\Gamma_{c}\right)$, the nondifferentiability constraint on $\bar{\lambda}_{f}$ can be simplified to

$$
\left|\bar{\lambda}_{f}\right| \leq s \text {, a.e. on } \Gamma_{c} \text {. }
$$

Note that from Lagrange multipliers update formula, we have

$$
\left[\boldsymbol{\lambda}_{f}^{k+1}\right]=\left[\boldsymbol{\lambda}_{f}^{k}\right]+r\left(\left[\mathbf{u}_{t}^{k}\right]-\left[\mathbf{p}_{f}^{k}\right]\right) .
$$

If $\left|\left[\boldsymbol{\lambda}_{f}^{k}\right]+r\left[\mathbf{u}_{t}\right]\right| \leq 2 s$, then $\left[\boldsymbol{p}_{f}\right]=0$ and

$$
\left[\boldsymbol{\lambda}_{f}^{k+1}\right]=\left[\boldsymbol{\lambda}_{f}^{k+1}\right]+r\left[\mathbf{u}_{t}^{k}\right]
$$

We deduce that $\left[\boldsymbol{\lambda}_{f}^{k+1}\right] / 2$ verifies (4.1).

If $\left|\left[\boldsymbol{\lambda}_{f}^{k}\right]+r\left[\mathbf{u}_{t}\right]\right| \geq 2 s$, then

$$
\left[\mathbf{p}_{f}^{k}\right]=\left[\mathbf{u}_{t}^{k}\right]+\frac{1}{r}\left[\boldsymbol{\lambda}_{f}^{k}\right]-\frac{2 s}{r} \frac{\left[\boldsymbol{\lambda}_{f}^{k}\right]+r\left[\mathbf{u}_{t}^{k}\right]}{\left|\left[\boldsymbol{\lambda}_{f}^{k}\right]+r\left[\mathbf{u}_{t}^{k}\right]\right|},
$$

that is

$$
r\left(\left[\mathbf{u}_{t}^{k}\right]-\left[\mathbf{p}_{f}^{k}\right]\right)=-\left[\boldsymbol{\lambda}_{f}^{k}\right]+2 s \frac{\left[\boldsymbol{\lambda}_{f}^{k}\right]+r\left[\mathbf{u}_{t}^{k}\right]}{\left|\left[\boldsymbol{\lambda}_{f}^{k}\right]+r\left[\mathbf{u}_{t}^{k}\right]\right|} .
$$

Substituting (4.3) into (4.2), we get

$$
\left[\boldsymbol{\lambda}_{f}^{k+1}\right]=2 s \frac{\left[\boldsymbol{\lambda}_{f}^{k}\right]+r\left[\mathbf{u}_{t}^{k}\right]}{\left|\left[\boldsymbol{\lambda}_{f}^{k}\right]+r\left[\mathbf{u}_{t}^{k}\right]\right|}
$$

and we deduce that (4.1) is valid for $\left[\boldsymbol{\lambda}_{f}^{k+1}\right] / 2$, for all $k \geq 0$.

Since the set $\left\{\mu \in L^{2}\left(\Gamma_{c}\right):|\mu| \leq s\right\}$ is convex, then the weak limit of the sequence $\left\{\left[\boldsymbol{\lambda}_{f}^{k}\right] / 2\right\}$, i.e. $\left[\boldsymbol{\lambda}_{f}\right] / 2$, satisfies the inequality (4.1). Finally we conclude that, $\left[\boldsymbol{\lambda}_{f}^{k}\right] / 2 \rightarrow$ $\left[\boldsymbol{\lambda}_{f}\right] / 2=\bar{\lambda}_{f}$. 


\section{$5 \quad$ Numerical experiments}

Algorithms UBR-DDM was implemented in Matlab 7 using piecewise linear finite element and vectorized codes [22]. The test problems used are designed to illustrate the behavior of the algorithm more than to model contact actual phenomena. Nondimensionalized units are used and the deformed configurations are plotted with a magnification of the displacement.

Since the matrices of the linear elasticity subproblems (3.8) do not change during the iterative process, a Cholesky factorization is performed once and for all in the initialization step. Then, in the rest of the iterative process, the solution of the linear elasticity subproblems reduces to foreward/backward substitutions. To reduce fill-in, the Cholesky factorization is done after columns and rows permutation with the Matlab function symamd.

In all numerical experiments reproted in this section, the stopping criterion is

$$
\left\|\mathbf{u}_{h}^{k}-\mathbf{u}_{h}^{k-1}\right\|_{0, \Omega}^{2}+\left\|\mathbf{p}_{h}^{k}-\mathbf{p}_{h}^{k-1}\right\|_{0, \Gamma_{c}}^{2}<10^{-12}\left(\left\|\mathbf{u}_{h}^{k}\right\|_{0, \Omega}^{2}+\left\|\mathbf{p}_{h}^{k}\right\|_{0, \Gamma_{c}}^{2}\right)
$$

The penalty parameter is $r=\min \left(E^{1}, E^{2}\right)$ in $\S 5.1$ and $r=\max \left(E^{1}, E^{2}\right)$ in $\S 5.2$.

\subsection{Example 1: Hertz problem}

To illustrate the numerical behavior of Algorithm UBR-DDM, a Hertz contact problem is considered, see e.g. [19]. The problem consist of an infinitely long elastic cylinder $\Omega^{1}$ (radius $\left.R=8, E^{1}=2 \times 10^{3}, \nu^{1}=0.3\right)$ resting on an elastic foundation $\Omega^{2}\left(E^{1}=10^{4}\right.$, $\left.\nu^{1}=0.4\right)$. The cylinder is subjected to a uniform load, along its top, of intensity $P=1600$. The friction coefficient is $\nu_{f}=0.6$. Taking into account the symmetry of the problem, we restrict our study to the subdomains

$$
\begin{aligned}
& \Omega_{1}=\left\{\left(x_{1}, x_{2}\right) \mid 0 \leq x_{1} \leq 8, R-\sqrt{R^{2}-x_{1}^{2}} \leq x_{2} \leq 8\right\}, \\
& \Omega_{1}=\left\{\left(x_{1}, x_{2}\right) \mid 0 \leq x_{1} \leq 8,-4 \leq x_{2} \leq 0\right\}
\end{aligned}
$$

with suitable boundary conditions. The contact surfaces are

$$
\Gamma_{c}^{1}=\left\{\left(x_{1}, x_{2}\right) \mid 0 \leq x_{1} \leq 4, x_{2}=R-\sqrt{R^{2}-x_{1}^{2}}\right\} \text { and } \Gamma_{c}^{2}=(0,4) \times\{0\}
$$

Their common projection is $\Gamma_{c}=(0,4) \times\{0\}$ and the normalized gap is $g\left(x_{1}\right)=x_{2}$. For the frictionless problem we will compare our numerical contact pressure with the analytical contact pressure due to Hertz (see e.g. [14, 18]). For the problem with Tresca friction, we set $s(x)=\nu_{f}|p(x)|$, where $p$ is the analytical contact pressure. 


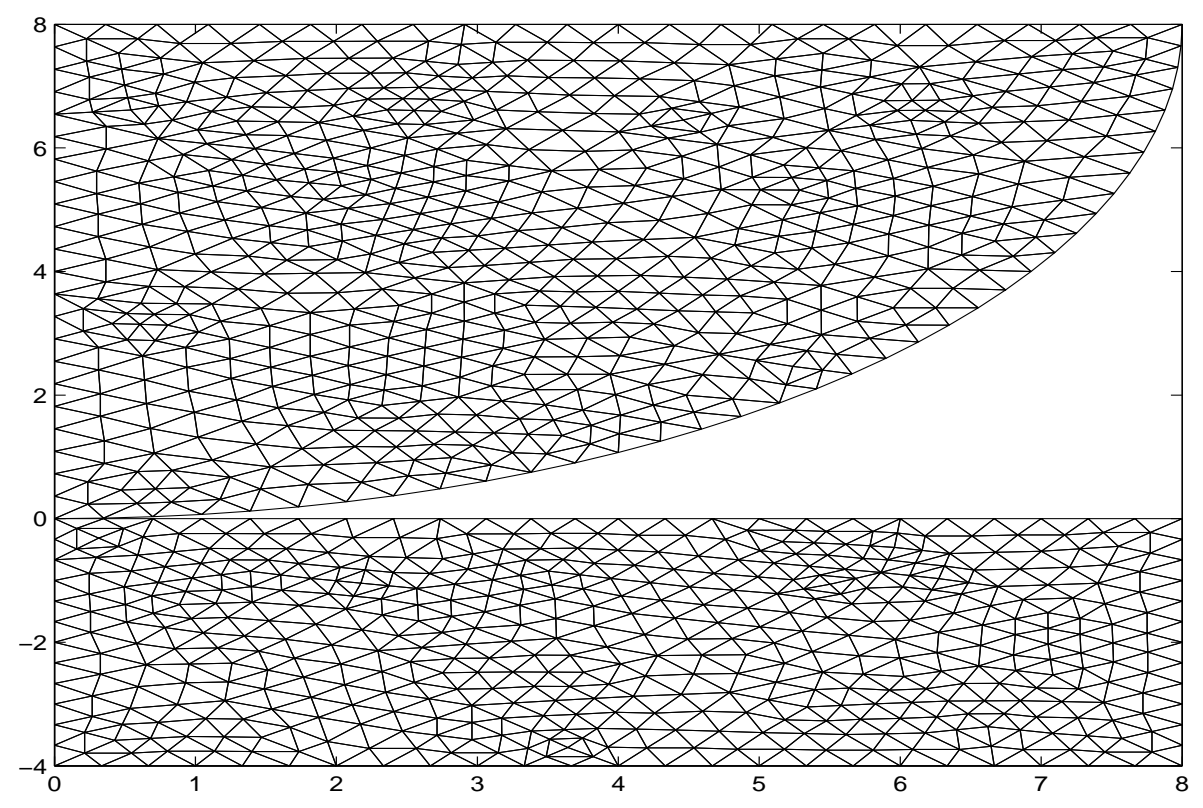

Figure 1: Mesh sample for a Hertz contact problem

\subsubsection{Matching meshes}

We first study the Hertz problem with the node-on-node contact condition. In this case, it is necessary that the nodes opposite to one another on $\Gamma_{c}^{1}$ and $\Gamma_{c}^{2}$ have the same $x_{1}$ coordinate. The subdomains $\Omega^{1}$ and $\Omega^{2}$ are discretized using nonuniform meshes consisting of 649 and 489 nodes, respectively, with 13 nodes on $\Gamma_{c}$, as shown in Figure 1.

Applying to this problem, Algorithm UBR-DDM stops after 40 iterations in the frictionless case and 32 in the case with friction. Figure 2 shows the deformed configuration in frictionless case. The grey tones visualize the Von Mises effective stress distribution in $\Omega^{1}$ and $\Omega^{2}$. In Figures 3-4 we compare the numerical (interface) stress, obtained with Algorithm UBR-DDM, and the analytical Hertz solution. We observe a quite good agreement with the Hertz solution even though a coarse mesh is used.

To study the scalability of the algorithm, the initial mesh is uniformly refined successively to produce meshes with $2513 / 1881,9889 / 7377,39233 / 29217$ nodes on $\Omega^{1} / \Omega^{2}$ with 25, 49 and 97 nodes on $\Gamma_{c}$. Table 1 shows the behavior of the algorithm. We can notice that the number of iterations is virtually independent of the mesh size, i.e. the algorithm is scalable.

\subsubsection{Nonmatching meshes}

In the case the meshes do not match on $\Gamma_{c}$, we have two meshes $\Gamma_{c h_{1}}$ and $\Gamma_{c h_{2}}$ for $\Gamma_{c}$. The mesh $\Gamma_{c h_{1}}\left(\right.$ resp. $\left.\Gamma_{c h_{2}}\right)$ is induced by $\Omega_{h}^{1}$ (resp. $\Omega_{h}^{2}$ ). The contact condition $(2.9)$ is then taken into account using a node-to-segment condition $[19,29,30,31]$. We use projection procedures to compute the auxiliary interface unknowns while the rest of the 


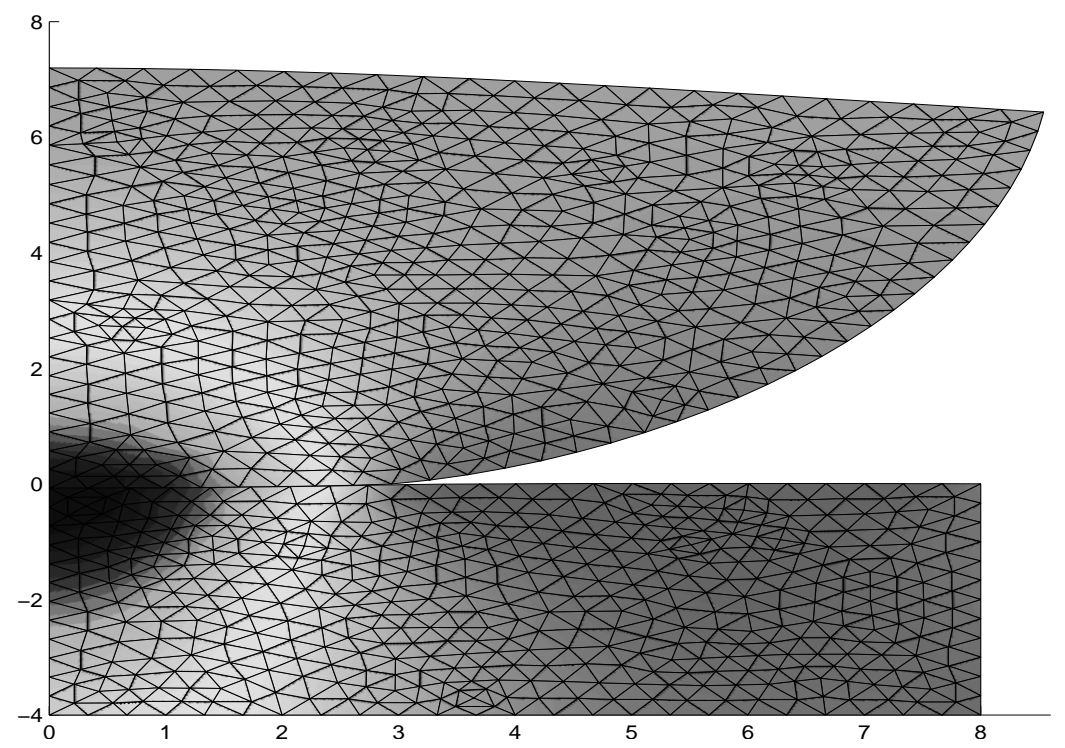

Figure 2: Deformed configuration and Von Mises effective stress for a Hertz problem without friction

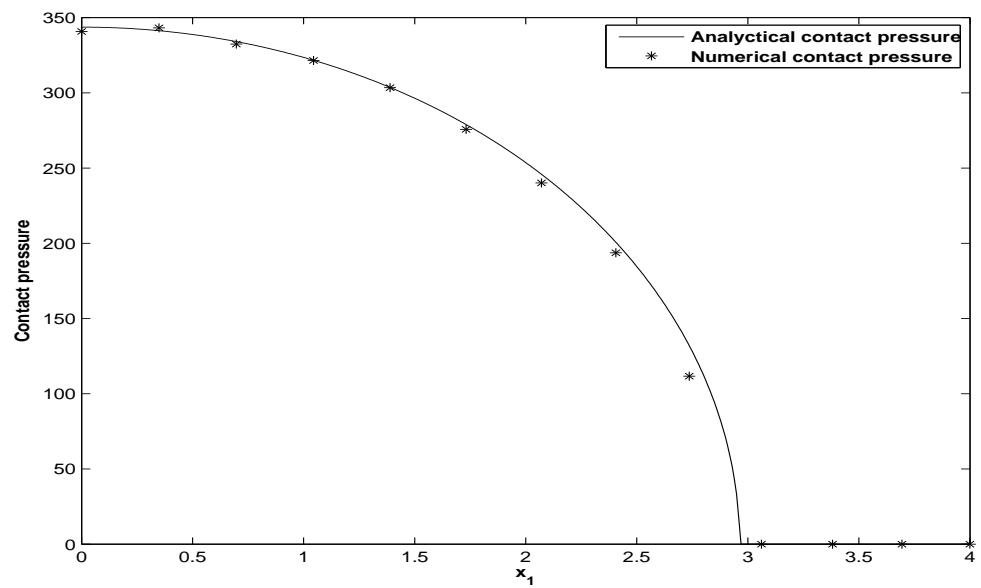

Figure 3: Contact pressure distribution for a Hertz problem without friction 


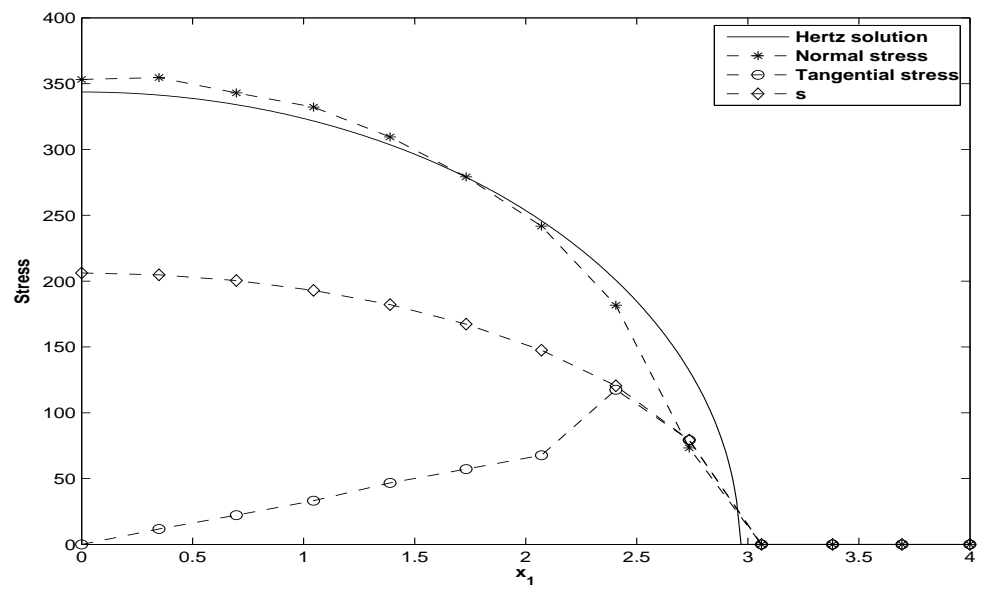

Figure 4: Stress distributions for a Hertz problem with Tresca friction

\begin{tabular}{|c|cc|}
\hline & \multicolumn{2}{|c|}{ Number of Iterations } \\
\hline Interface nodes & Frictionless case & Friction case \\
\hline 13 & 40 & 32 \\
25 & 48 & 42 \\
49 & 48 & 45 \\
97 & 46 & 42 \\
\hline
\end{tabular}

Table 1: Performances of Algorithm UBR-DDM on Hertz problem with matching meshes 
algorithm remains unchanged. For instance, for the auxiliary variable $\boldsymbol{p}_{c}$, we replace (3.13) by the following formulas

$$
\begin{aligned}
& p_{c h}^{1, k}=u_{n h}^{1, k}+\frac{1}{r} \lambda_{c h}^{1, k}-\frac{1}{2 r}\left(\lambda_{c h}^{1, k}-\pi_{1}\left(\lambda_{c h}^{2, k}\right)+r\left(u_{n h}^{1, k}-\pi_{1}\left(u_{n h}^{2, k}\right)-g\right)\right)^{+} \\
& p_{c h}^{2, k}=u_{n h}^{2, k}+\frac{1}{r} \lambda_{c h}^{2, k}-\frac{1}{2 r}\left(\pi_{2}\left(\lambda_{c h}^{1, k}\right)-\lambda_{c h}^{2, k}+r\left(\pi_{2}\left(u_{n h}^{1, k}\right)-u_{n h}^{2, k}-g\right)\right)^{+}
\end{aligned}
$$

where $\pi_{\alpha}(\cdot)$ stands for the projection onto $\Gamma_{c h_{\alpha}}$. We then obtain a symmetric nodeto-segment contact condition. The so-called "locking" phenomenon (see e.g. [19]) is avoided since the node-on-segment condition is applied in a symmetrical way.

For the numerical behavior of the algorithm, we use a sequence of nonmatching meshes with $524 / 253,2023 / 961,7949 / 3745$ and $31513 / 14785$ nodes on $\Omega_{h}^{1} / \Omega_{h}^{2}$; and $11 / 9,21 / 17,41 / 33$ and $81 / 65$ nodes on $\Gamma_{c h_{1}} / \Gamma_{c h_{2}}$. Figures $5-6$ show the undeformed and deformed configurations with the coarse mesh. Figures 7-8 depict the stress distributions on $\Gamma_{c}$. The results are in accordance with the ones of the node-to-node contact condition. We report in Table 2 the performance of the algorithm with nonmatching meshes. We can notice that the number of iterations required for convergence is virtually independent of the mesh size.

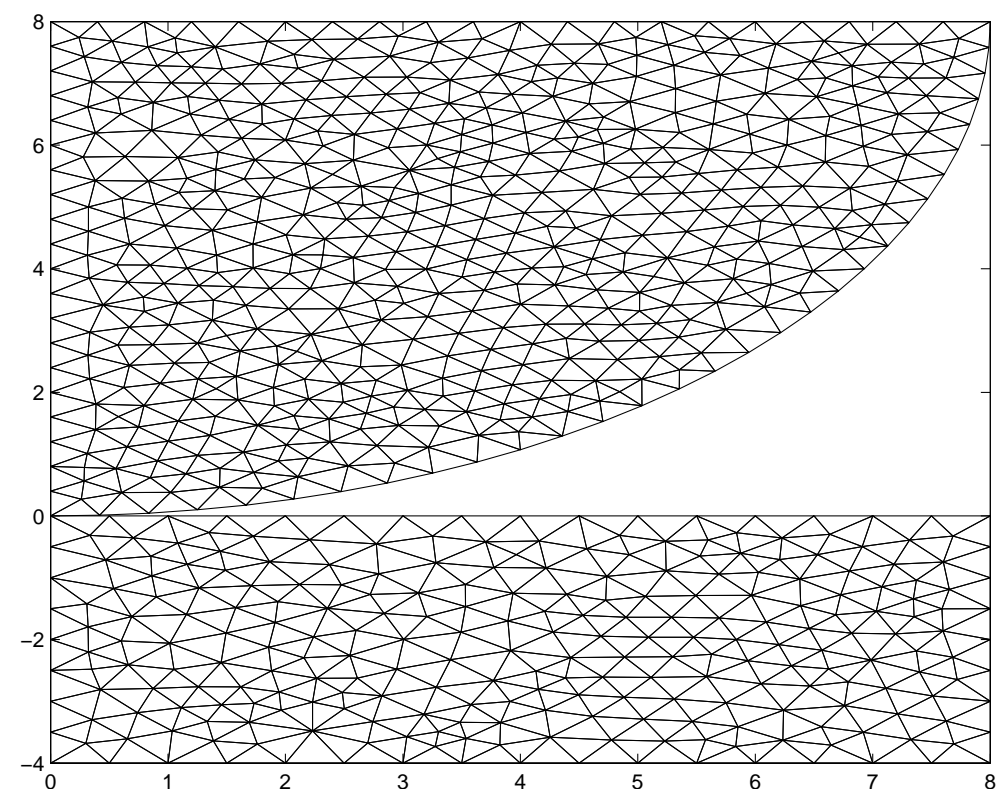

Figure 5: Hertz contact problem with nonmatching meshes: undeformed configuration 


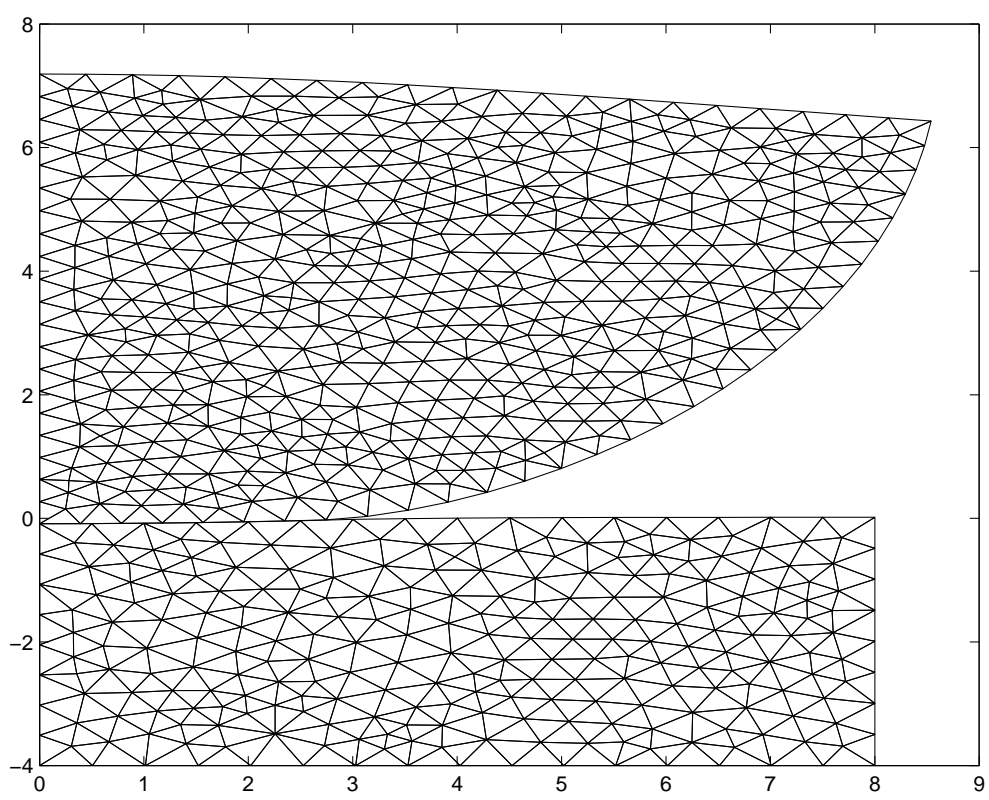

Figure 6: Hertz contact problem with nonmatching meshes: deformed configuration (frictionless case)

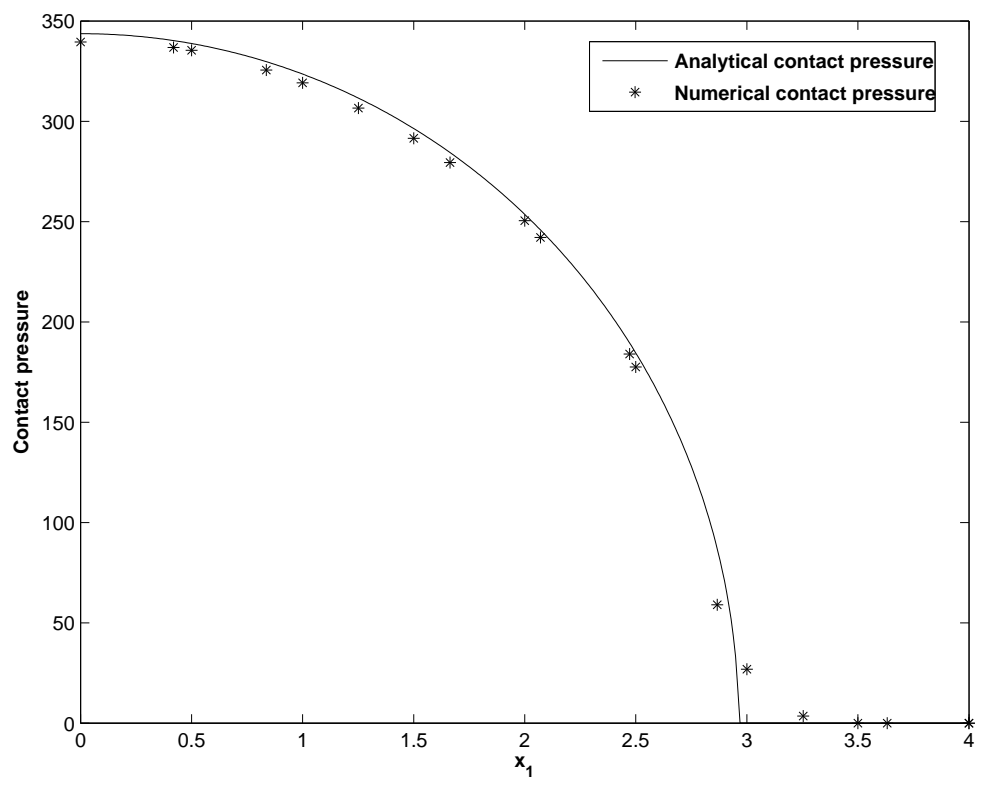

Figure 7: Hertz contact problem with nonmatching meshes: contact pressure (frictionless case) 


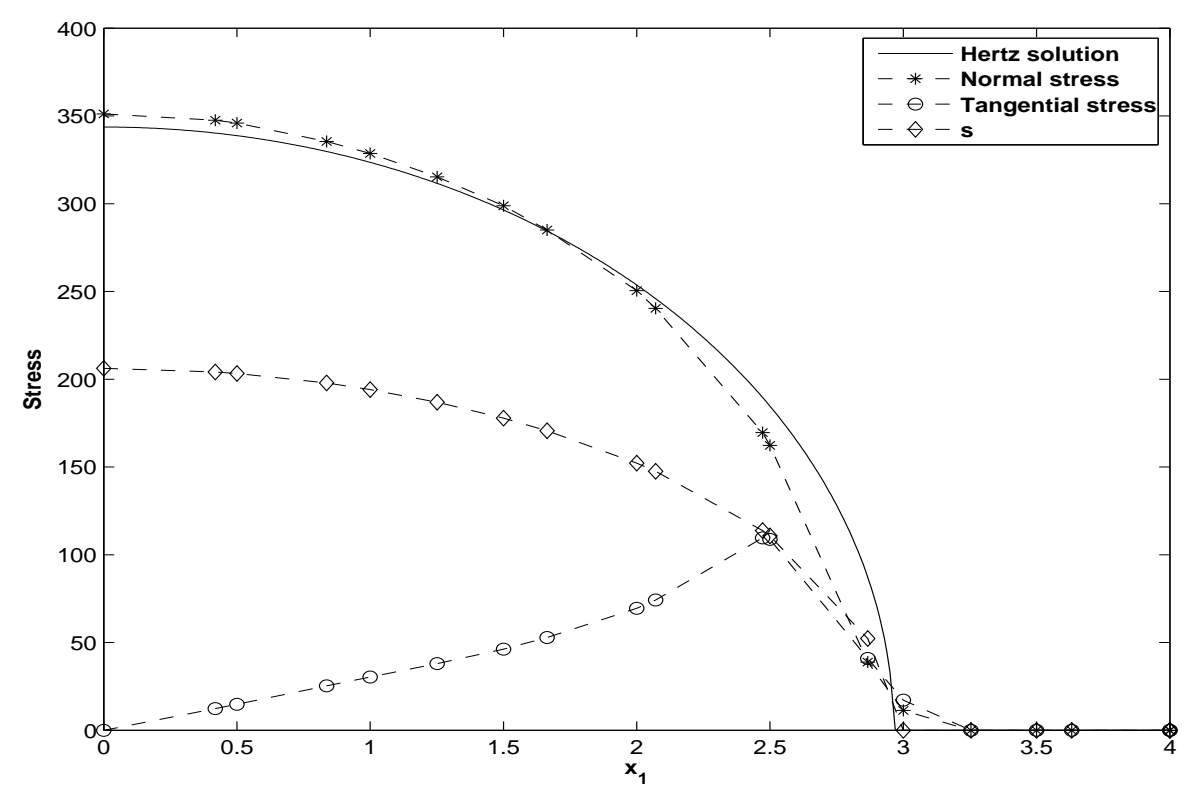

Figure 8: Hertz contact problem with nonmatching meshes: Stress distributions on $\Gamma_{c}$

\begin{tabular}{|c|cc|}
\hline & \multicolumn{2}{|c|}{ Number of iterations } \\
\hline Interface nodes on $\Gamma_{c h_{1}} / \Gamma_{c h_{1}}$ & Frictionless case & Friction case \\
\hline $11 / 9$ & 29 & 35 \\
$21 / 17$ & 30 & 39 \\
$41 / 33$ & 31 & 41 \\
$81 / 65$ & 32 & 38 \\
\hline
\end{tabular}

Table 2: Performances of Algorithm UBR-DDM on Hertz problem with nonmatching meshes 


\subsection{Example 2}

This example is derived from [16], see Figure 9. We consider the contact problem beetwin two rectangular blocks

$$
\begin{aligned}
& \Omega^{1}=(0,1)^{2}, \Gamma_{D}^{1}=\{1\} \times(0,1), \Gamma_{N}^{1}=(0,1) \times\{1\}, \boldsymbol{g}^{1}=\left(\begin{array}{c}
0 \\
-100
\end{array}\right) \\
& \Omega^{2}=(0,1) \times(-1,0), \Gamma_{D}^{2}=(0,1) \times\{-1\} \cup\{1\} \times(-1,0) .
\end{aligned}
$$

The elastic constants are $E^{1}=13000, \nu_{1}=0.2, E^{2}=30000$ and $\nu_{2}=0.2$. The friction coefficient is $\nu_{f}=0.3$. For this problem, we have

$$
\Gamma_{c}^{1}=\Gamma_{c}^{2}=\Gamma_{c}=(0,1) \times\{0\} .
$$

The exact solution of this contact problem is a uniform $\sigma_{22}$ field of value -100 for the frictionless case. For the friction case we set $s=100 \nu_{f}$.

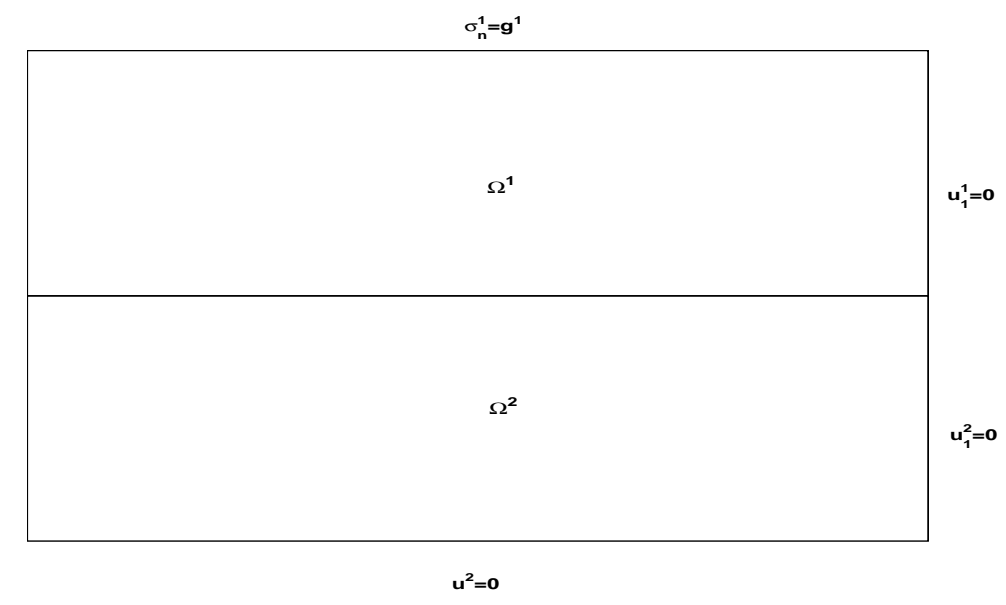

Figure 9: Geometry of the problem

\subsubsection{Matching meshes}

We first run the Matlab code with a uniform mesh of size 1/8 on the frictionless problem. Figure 10 shows the deformed configuration and the distribution of the vertical component of the stress $\sigma_{22}$. The color bar shows that the value of $\sigma_{22}$ is about -100 within both bodies. Figure 11 depicts the deformed configuration for the case with friction. We notice that the contact beetwin both blocks is in "stick" mode. Figure 12 shows the stress distributions on $\Gamma_{c}$ and confirms the "stick" mode since we have $\sigma_{t}<s=\nu_{f}\left|\sigma_{n}\right|$ on $\Gamma_{c}$.

For the numerical behavior of the algorithm, the mesh of size $1 / 8$ is successively refined to produce meshes with size $1 / 16,1 / 32,1 / 64$ and 1/128. The performances of Algorithm UBR-DDM is reported in Table 3. We notice again than the number of iterations is virtually independent of the mesh size. 


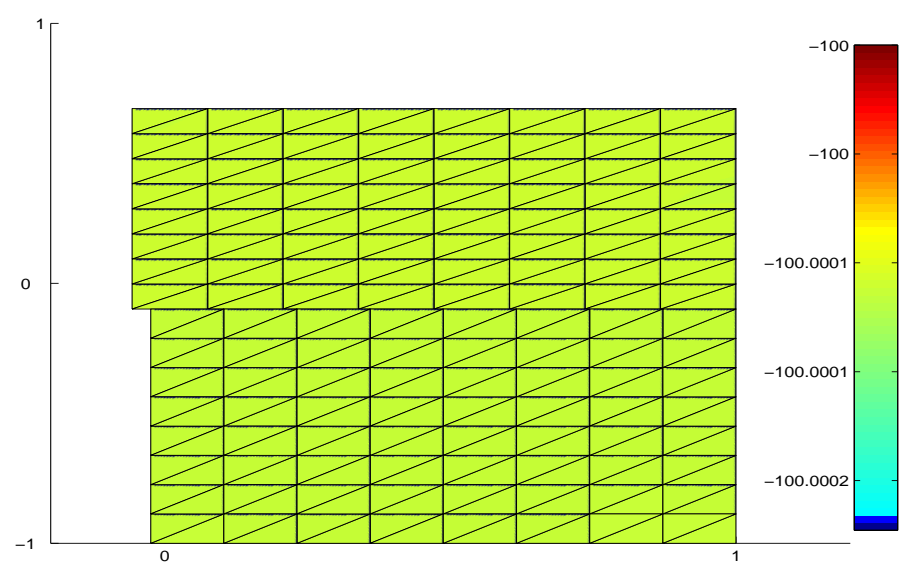

Figure 10: Deformed configuration and $\sigma_{22}$ distribution for the frictionless case

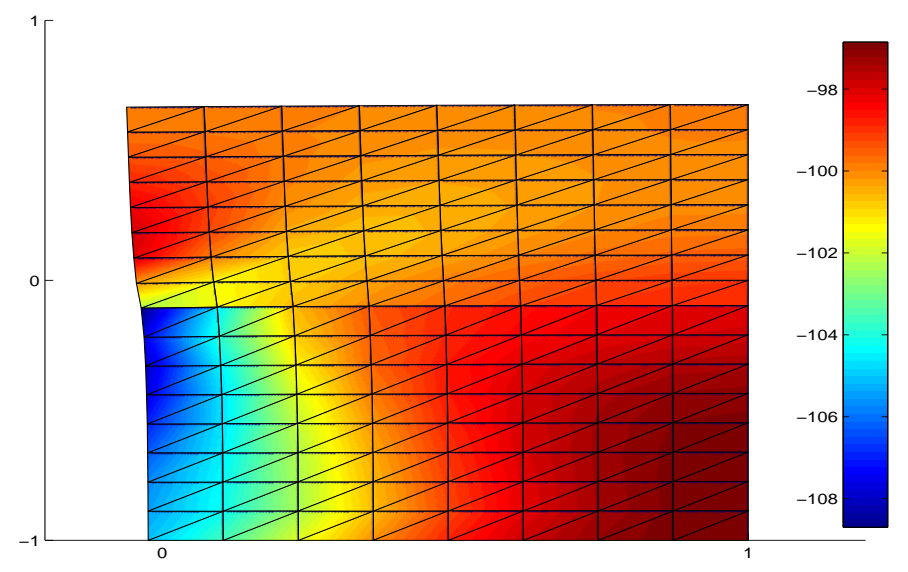

Figure 11: Deformed configuration and $\sigma_{22}$ distribution for the friction case

\begin{tabular}{|c|cc|}
\hline & \multicolumn{2}{|c|}{ Number of iterations } \\
\hline Mesh size & Frictionless case & Friction case \\
\hline $1 / 8$ & 20 & 42 \\
$1 / 16$ & 20 & 43 \\
$1 / 32$ & 20 & 37 \\
$1 / 64$ & 20 & 34 \\
$1 / 128$ & 20 & 34 \\
\hline
\end{tabular}

Table 3: Performances of Algorithm UBR-DDM with matching meshes 


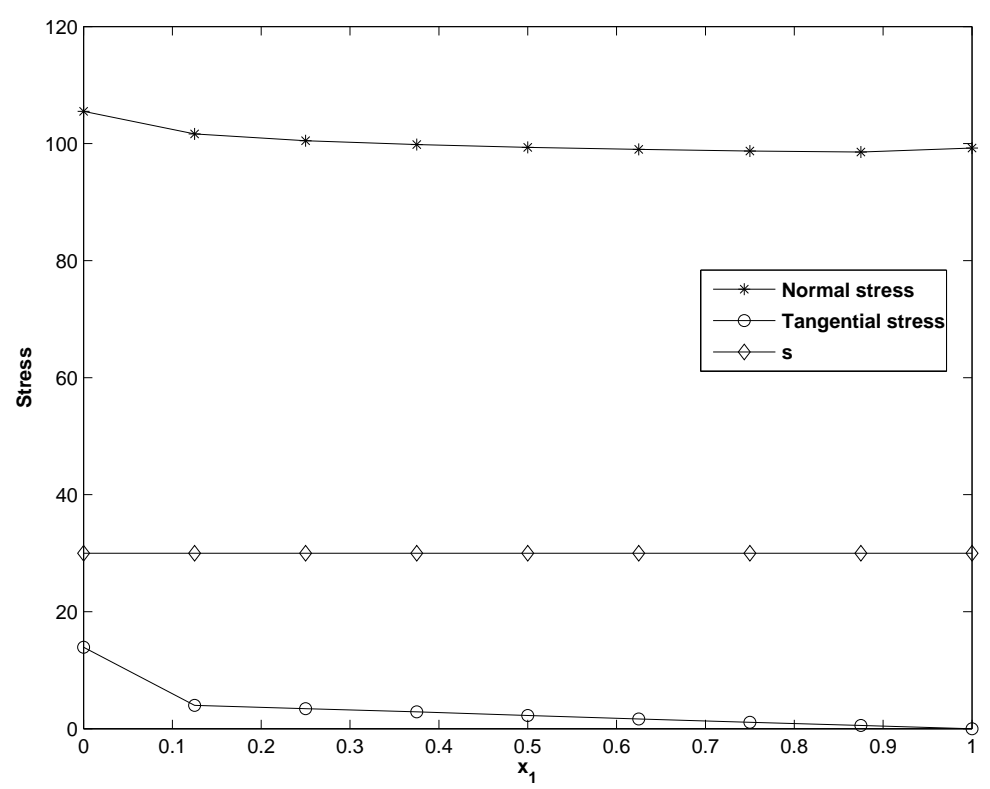

Figure 12: Stress distribution on $\Gamma_{c}$

\begin{tabular}{|c|cc|}
\hline & \multicolumn{2}{|c|}{ Number of iterations } \\
\hline Interface nodes on $\Gamma_{c h_{1}} / \Gamma_{c h_{1}}$ & Frictionless case & Friction case \\
\hline $9 / 5$ & 20 & 25 \\
$17 / 9$ & 20 & 30 \\
$33 / 17$ & 20 & 38 \\
$65 / 33$ & 20 & 41 \\
$129 / 65$ & 20 & 40 \\
\hline
\end{tabular}

Table 4: Performances of Algorithm UBR-DDM with nonmatching meshes 


\subsubsection{Nonmatching meshes}

We have also used Algorithm UBR-DDM with a sequence of nonmatching meshes. Figure 13 shows the deformed configuration (friction case), in accordance with Figure 11. We report in Table 4 the performace of Algorithm UBR-DDM. We notice again that the number of iterations is virtually independent of the mesh size.

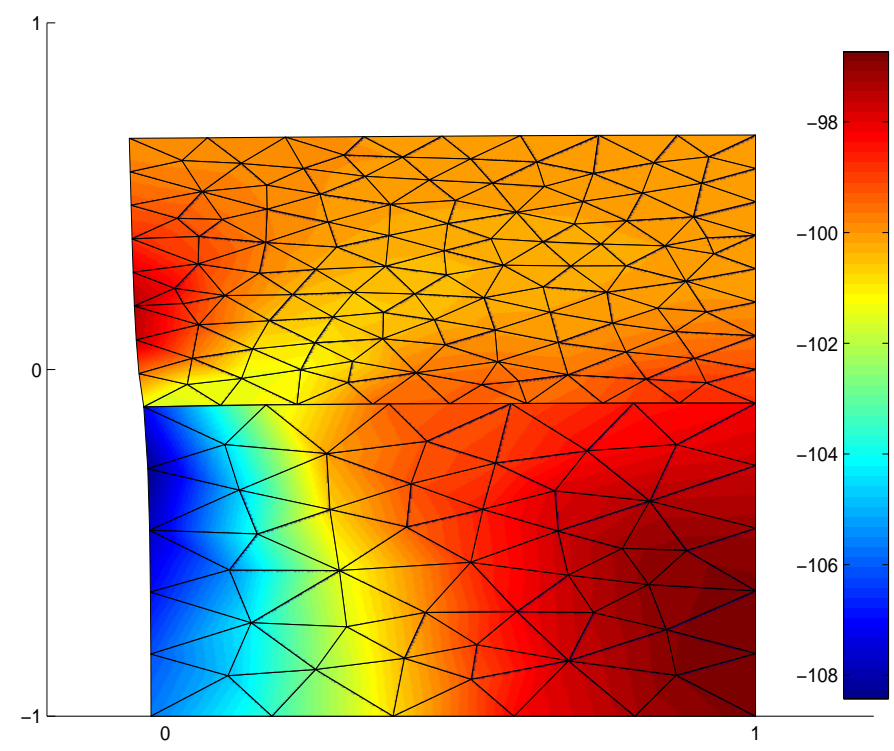

Figure 13: Deformed configuration and $\sigma_{22}$ distribution for the friction case and nonmatching meshes

\subsubsection{Three-dimensional case}

We now study the three-dimensional version of the problem. The contact surface becomes

$$
\Gamma_{c}^{1}=\Gamma_{c}^{2}=\Gamma_{c}=(0,1) \times(0,1) \times\{0\} .
$$

The exact solution is still a uniform $\sigma_{33}$ field of value -100 for the frictionless case. For the friction case we set $s(x)=100 \nu_{f}$ on $\Gamma_{c}$.

Figure 14 shows a uniform mesh sample of size $1 / 4$ while Figure 15 shows the deformed configuration in accordance with the two-dimensional results. We report in Table 5 the performances of the algorithm showing its scalability.

\section{Conclusion}

We have studied a new domain decomposition method for the two-body contact problem with Tresca friction. Numerical experiments have shown that the algorithm is scalable 


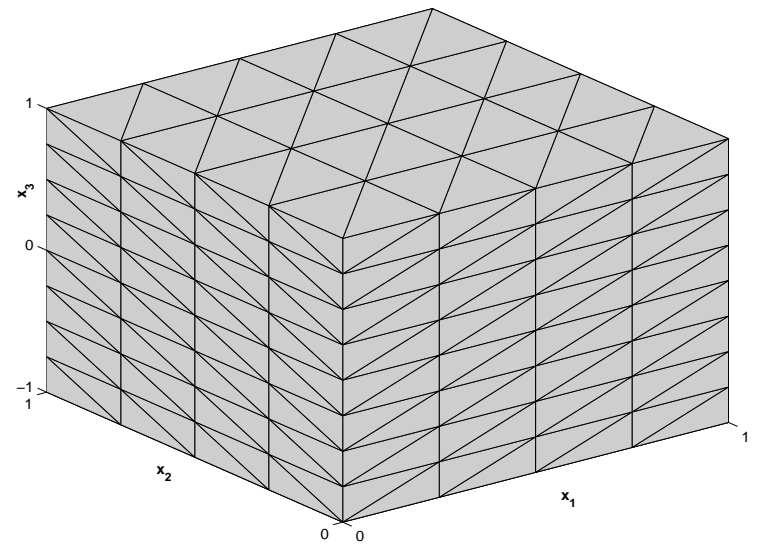

Figure 14: Mesh sample of size 1/4 for the three dimensional contact problem

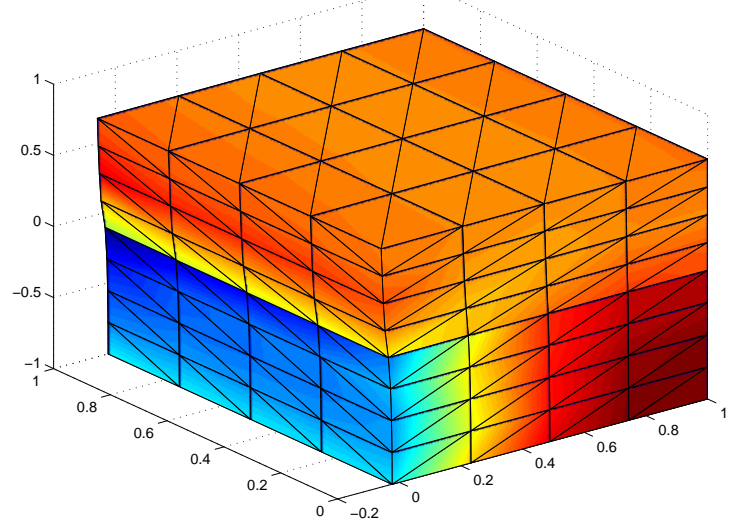

Figure 15: Deformed configuration and $\sigma_{33}$ distribution for the problem with friction

\begin{tabular}{|c|cc|}
\hline & \multicolumn{2}{|c|}{ Number of iterations } \\
\hline Mesh size & Frictionless case & Friction case \\
\hline $1 / 4$ & 20 & 34 \\
$1 / 8$ & 20 & 42 \\
$1 / 16$ & 20 & 43 \\
$1 / 32$ & 20 & 43 \\
\hline
\end{tabular}

Table 5: Performances of Algorithm UBR-DDM on a three-dimensional problem 
with matching or nonmatching meshes.

To reduce the size of the linear elasticity subproblems, the standard domain decomposition constraints can be added to the constrained optimization problem (3.1)-(3.3) for each subdomain. Using a three-field formulation, see e.g. [26, 27], we can derive a Uzawa block relaxation domain decomposition method with "small" subdomain problems.

\section{References}

[1] Bayada G., Sabil J. and Sassi T. Algorithme de Neumann-Dirichlet pour des problèmes de contact unilateral: Résultat de convergence. C. R. Acad. Sci. Paris, Ser. I, 335:381-386, 2002.

[2] Bayada G., Sabil J. and Sassi T. A Neumann-Neumann domain decomposition algorithm for the Signorini problem. Appl. Math. Lett., 17:1153-1159, 2004.

[3] Bertsekas D. Constrained Optimization and Lagrange Multipliers Methods. Academic Press, New York, 1982.

[4] Bertsekas D. Nonlinear Programming. Athena Scientific, Nashua, NH, 1999.

[5] Cea J. and Glowinski R. Sur des méthodes d'optimisation par relaxation. R.A.I.R.O., R-3:5-32, 1973.

[6] Dostál Z. and HorÁk D. Scalable FETI with optimal dual penalty for variational inequalities. Numer. Linear Algebra Appl., 11:455-472, 2004.

[7] Dostál Z., Gomes Neto F. A. M. and Santos S. A. Solution of contact problems by FETI domain decomposition with natural coarse space projection. Comput. Methods. Appl. Mech. Engrg., 190:1611-1627, 2000.

[8] Dostál Z., Haslinger J. and KuČERA R. Implementation of the fixed point method in contact problems with Coulomb friction based on a dual splitting type technique. J. Comput. Appl. Math., 140:245-256, 2002.

[9] Dureisseix D. and Farhat C. A numerically scalable domain decomposition method for solution of frictionless contact problems. Internat. J. Numer. Methods Engrg, 50:2643-2666, 2001).

[10] Ekland I. and Temam R. Convex Analysis and Variational Problems. Classics in Applied Mathematics. SIAM, Philadelphia, 1999.

[11] Farhat C. and Roux F. X. A method of finite element reearing and interconnecting and its parallel solution algorithm. Internat. J. Numer. Methods Engrg, 32:1205-1227, 1991.

[12] Fortin M. and Glowinski R. Augmented Lagrangian Methods: Application to the Numerical Solution of Boundary-Value Problems. North-Holland, Amsterdam, 1983. 
[13] Glowinski R. and Le Tallec P. Augmented Lagrangian and Operator-splitting Methods in Nonlinear Mechanics. Studies in Applied Mathematics. SIAM, Philadelphia, 1989.

[14] Goldsmith W. Impact. Edward Arnold, London, 1960.

[15] Haslinger J., Dostál Z. and KuČera R. On the splitting type algorithm for the numerical realization of the contact problems with Coulomb friction. Comput. Methods. Appl. Mech. Engrg., 191:2261-2281, 2002.

[16] Hild P. Numerical implementation of two noncoforming finite element methods for unilateral contact. Comput. Methods. Appl. Mech. Engrg., 184:99-123, 2000.

[17] Ito K. and Kunisch K. The augmented lagrangian method for equality and inequality constraints in Hilbert scpaces. Math. Program., 46:341-360, 1990.

[18] Johnson K.L. Contact Mechanics. Cambridge University Press, Cambridge, 1987.

[19] Kikuchi N. and Oden J.T. Contact problems in Elasticity: A Study of Variational Inequalities and Finite Element Methods. Studies in Applied Mathematics. SIAM, Philadelphia, 1988.

[20] Kоко J. An optimization based domain decomposition method for a two-body contact problem. Numer. Funct. Anal. Optim., 24(5-6):587-605, 2003.

[21] Koко J. Lagrange multiplier based domain decomposition methods for a nonlinear sedimentary basin problem. Comput. Geosci., 2007. To appear.

[22] Kоко J. Vectorized Matlab codes for two-dimensional linear elasticity. Scientific Programming, 2007. To appear.

[23] Kosior F., Guyot N. and Maurice G. Analysis of frictional contact problem using boundary element method and domain decomposition method. Internat. J. Numer. Methods Engrg., 46:65-82, 1999.

[24] Licht C., Pratt E. and Raous M. Remarks on a numerical method for unilateral contact including friction. In Unilateral problems in structural analysis, volume 101 of Internat. Ser. Numer. Math., pages 129-144. Birkh'auser, Nasel, 1991.

[25] Luenberger D. Linear and Nonlinear Programming. Addison Wesley, Reading, MA, 1989.

[26] Magoulès F. and Roux F.-X. Lagrangian formulation of domain decomposition methods: A unified theory. Appl. Math. Modelling, 30:593-615, 2006.

[27] Quarteroni A. and Valli A. Domain Decomposition Methods for Partial Differential Equations. Oxford University Press, 1999. 
[28] SchöBerL J. Solving the Signorini problem on the basis of domain decomposition techniques. Computing, 60:323-244, 1998.

[29] Wriggers P. and Simo J.C. A note on tangent stiffness for fully nonlinear contact problems. Comm. in Appl. Num. Methods, 1:199-203, 1985.

[30] Zavarise G. and Wriggers P. A segment-to-segment contact strategy. Mathl. Comput. Modelling, 28:497-515, 1998.

[31] Zavarise G., Wriggers P., Stein E. and Schrefler B.A. Real contact mechanisms and finite element formulation-A coupled thermomechanical approach. Internat. J. Numer. Methods Engrg, 35:767-785, 1992. 